\title{
FOURIER SERIES IN SEVERAL VARIABLES
}

\author{
VICTOR L. SHAPIRO ${ }^{1}$
}

0. Preface. This article is a survey of certain aspects of the theory of multiple Fourier and trigonometric series. It is by no means meant to be a complete survey; for example, it is practically disjoint with the material covered on the subject in Zygmund's book [38, Chapter 17.].

There are eight sections to this survey. $\S 1$ is the introduction. $\$ 2$, $\$ 3$, and $\$ 4$ are expository in the sense that the main theorems in each section are proved. $\S 5, \S 6$, and $\S 7$ are descriptive. $\S 8$ consists of two bibliographies, a bibliography for the survey itself, and a general bibliography.

$\$ 2$ deals with the now classical theory of the Bochner-Riesz summability of multiple Fourier series and the Abel summability of multiple Fourier series. $\$ 3$ presents Bochner's counter-example for the critical index in summability theory in considerable detail. $\$ 4$ is concerned with the uniqueness of multiple trigonometric series and proves the main theorem in the subject so far, i.e. uniqueness under Abel summability (due to the present author). $\$ 5$ describes some results in conjugate multiple Fourier series defined by means of the Calderón-Zygmund kernel and related topics, i.e. analyticity in several variables. $\$ 6$ deals with the Riemannian theory of multiple trigonometric series. $\$ 7$ describes some applications to geometric integration theory and potential theory.

1. Introduction. Operating in $k$-dimensional Euclidean space, $E_{k}$, $k \geqq 2$, we shall use the following notation:

$$
\begin{gathered}
x=\left(x_{1}, \cdots, x_{k}\right), \quad y=\left(y_{1}, \cdots, y_{k}\right), \\
\alpha x+\beta y=\left(\alpha x_{1}+\beta y_{1}, \cdots, \alpha x_{k}+\beta y_{k}\right), \\
(x, y)=x_{1} y_{1}+\cdots+x_{k} y_{k}, \quad|x|=(x, x)^{1 / 2}, \\
m=\left(m_{1}, \cdots, m_{k}\right), \quad(m, x)=m_{1} x_{1}+\cdots+m_{k} x_{k} .
\end{gathered}
$$

With $f(x)$ a function in $L^{1}$ on $T_{k}$, the $k$-dimensional torus

$$
\left\{x ;-\pi<x_{j} \leqq \pi, j=1, \cdots, k\right\}
$$

and $m$ an integral lattice point, we shall designate the series

1 This work was supported by the Air Force Office of Scientific Research and by National Science Foundation grant NSF-G16428 to the American Mathematical Society; received by the editors August 27, 1963. 


$$
\sum_{m} f(m) e^{i(m, x)}
$$

by $S[f]$ and call it the Fourier series of $f$ where

$$
\hat{f}(m)=(2 \pi)^{-k} \int_{T_{k}} e^{-i(m, x)} f(x) d x .
$$

It is the purpose of this paper to give a survey of some of the known results concerning $S[f]$ and concerning trigonometric series of the form $\sum_{m} a_{m} e^{i(m, x)}$.

With $\Delta=\partial^{2} / \partial x_{1}^{2}+\cdots+\partial^{2} / \partial x_{k}^{2}$, we observe that $\Delta e^{i(m, x)}$ $=-|m|^{2} e^{i(m, x)}$. Consequently from an eigenvalue point of view, the first natural question to ask concerning $S[f]$ is "In what manner, does the series

$$
\sum_{n=1}^{\infty}\left(\sum_{|m|^{2}=n} f(m) e^{i(m, x)}\right)
$$

approximate $f$ ?" Bearing in mind the classical counter-examples of Fejer and Lebesgue [37, Chapter 8] concerning the one-dimensional Fourier series of continuous functions, we see that the answer to the above question should be phrased in terms of some summability method. The two most natural methods are those of Bochner-Riesz and Abel. In particular, we shall say that $S[f]$ is Bochner-Riesz summable of order $\alpha$, henceforth designated by $(B-R, \alpha)$, to $f(x)$ if

$$
\lim _{R \rightarrow \infty} \sum_{|m| \leq R} f(m) e^{i(m, x)}\left(1-|m|^{2} / R^{2}\right)^{\alpha}=f(x) .
$$

Bochner-Riesz summability plays the same role for multiple Fourier series that Cesaro summability plays for one-dimensional Fourier series. For further comments on summability methods for multiple Fourier series, see the classical paper of Bochner [3].

We say $S[f]$ is Abel summable to $f(x)$ if

$$
\lim _{i \rightarrow 0+} \sum_{m} f(m) e^{i(m, x)-|m| t}=f(x) .
$$

The reason for calling this method of summability Abel summability is motivated by the fact that the series

$$
\sum_{m} f(m) e^{i(m, x)-|m| t}
$$

is harmonic in upper $E_{k+1}$, i.e. in the variables $(x, t)$, for $t>0$.

2. Summability. Given $f$ in $L^{1}$ on $T_{k}$ (and real-valued unless explicitly stated otherwise), we shall say that $f$ is extended by periodic- 
ity to $E_{k}$ if $f$ is defined in all of $E_{k}$ and $f$ is periodic of period $2 \pi$ in each variable. Letting $B(x, r)$ represent the open $k$-ball with center $x$ and radius $r$ and letting $|B(x, r)|$ represent the $k$-dimensional volume of $B(x, r)$, i.e. $|B(x, r)|=2 \pi^{k / 2} r^{k} / k \Gamma(k / 2)$, the first theorem we prove is the following [3, p. 189];

Theorem 1. Let $f$ be in $L^{1}$ on $T_{k}$ and extended by periodicity to all of $E_{k}$, and let $S[f]=\sum_{m} \hat{f}(m) e^{i(m, x)}$. Set

$$
\sigma_{R}^{\alpha}(f, x)=\sum_{|m| \lesssim R} \hat{f}(m) e^{i(m, x)}\left(1-|m|^{2} / R^{2}\right)^{\alpha} .
$$

Suppose that $\left|B\left(x_{0}, h\right)\right|^{-1} \int_{B\left(x_{0}, h\right)}\left|f(x)-f\left(x_{0}\right)\right| d x \rightarrow 0$ as $h \rightarrow 0$. Then

$$
\lim _{R \rightarrow \infty}{ }_{\sigma_{R}}^{\alpha}\left(f, x_{0}\right)=f\left(x_{0}\right) \quad \text { for } \alpha>(k-1) / 2 .
$$

It is to be noted that this theorem implies that

$$
\underset{\sigma_{R}}{\alpha}(f, x) \rightarrow f(x)
$$

almost everywhere.

The standard technique for proving theorems of this nature is to first establish the analogous result for multiple Fourier integrals and then proceed by some form of the well-known Poisson summation formula [37, p. 68] to multiple Fourier series. We shall establish Theorem 1 in precisely this manner.

If $g$ is in $L^{1}$ on $E_{k}$, we shall designate the Fourier transform of $g$ by $\hat{g}$ and define $\hat{g}$ in a manner analogous to the Fourier coefficient of a function on $T_{k}$, i.e. $\hat{g}(u)=(2 \pi)^{-k} \int_{E_{k}} e^{-i(x, u)} g(x) d x$.

The first lemma we prove is the following:

Lemma 1. Let $g$ be in $L^{1}$ on $E_{k}$. Set

$$
\tau_{R}^{\alpha}(g, x)=\int_{B(0, R)} e^{i(x, u)} \hat{g}(u)\left(1-|u|^{2} / R^{2}\right)^{\alpha} d u .
$$

Suppose that $\left|B\left(x_{0}, h\right)\right|^{-1} \int_{B\left(x_{0}, h\right)}\left|g(x)-g\left(x_{0}\right)\right| d x \rightarrow 0$ as $h \rightarrow 0$. Then

$$
\lim _{R \rightarrow \infty} \tau_{R}^{\alpha}\left(g, x_{0}\right)=g\left(x_{0}\right) \quad \text { for } \alpha>(k-1) / 2 .
$$

(For a good introduction to the theory of multiple Fourier integrals, see [7, Chapter 2].)

We first observe from an iteration of well-known 1-dimensional theorems and from Lebesgue's dominated convergence theorem that 
the above lemma is clear for the special case $g(x)=e^{-\left|x-x_{0}\right|^{2}}$. Consequently, with no loss in generality, we can assume from the start that $g\left(x_{0}\right)=0$.

Next we observe from Fubini's theorem and the definition of $\hat{g}$ that

(1) $\quad \stackrel{\alpha}{\tau_{R}(g, x)}=(2 \pi)^{-k} \int_{E_{k}} g(y) d y \int_{B(0, R)} e^{i(x-y, u)}\left(1-|u|^{2} / R^{2}\right)^{\alpha} d u$.

Now it turns out that we can represent the inner integral on the right side of (1) in terms of Bessel functions. In particular, we observe from $[33$, p. 60$]$ and many other places that

$$
J_{\nu}(z)=\frac{z^{\nu}}{2^{\nu-1} \Gamma(\nu+1 / 2) \Gamma(1 / 2)} \int_{0}^{\pi / 2} \cos (z \cos \theta)(\sin \theta)^{2 \nu} d \theta
$$

$$
\text { for } \nu>-\frac{1}{2}
$$

and

(3) $J_{\mu+\nu+1}(z)=\frac{z^{\nu+1}}{2^{\nu} \Gamma(\nu+1)} \int_{0}^{\pi / 2} J_{\mu}(z \sin \theta)(\sin \theta)^{\mu+1}(\cos \theta)^{2 \nu+1} d \theta$

for $\mu>-1$ and $\nu>-1$.

We shall use (3) in the following form:

$$
\int_{0}^{t} J_{\mu}(s) s^{\mu+1}\left[1-s^{2} / t^{2}\right]^{\nu} d s=2^{\nu} \Gamma(\nu+1) J_{\mu+\nu+1}(t) t^{\mu+1-\nu} \quad \text { for } t>0 .
$$

Next, designating the inner integral in (1) by $(2 \pi)^{k} H_{R}^{\alpha}(x-y)$, we see, using spherical coordinates in $E_{k}$ (see [13, Chapter 11]) and letting $\omega_{k-1}$ designate the $(k-1)$-dimensional volume of the unit $(k-1)$ sphere, i.e. $\omega_{k-1}=2 \pi^{k / 2} / \Gamma(k / 2)$ and $\omega_{0}=2$, that by (2)

$$
\begin{aligned}
(2 \pi)^{k} H_{R}^{\alpha}(x)= & \omega_{k-2} \int_{0}^{R}\left(1-r^{2} / R^{2}\right)^{\alpha} r^{k-1}\left[\int_{0}^{\pi} e^{i r|x| \cos \theta}(\sin \theta)^{k-2} d \theta\right] d r \\
= & 2 \omega_{k-2} \int_{0}^{R}\left(1-r^{2} / R^{2}\right)^{\alpha} r^{k-1} \\
& \cdot\left[\int_{0}^{\pi / 2} \cos (r|x| \cos \theta)(\sin \theta)^{k-2} d \theta\right] d r \\
= & 2^{(k-2) / 2} \Gamma[(k-1) / 2] \Gamma(1 / 2) \omega_{k-2} \\
& \cdot \int_{0}^{R}\left\{\left(1-r^{2} / R^{2}\right)^{\alpha} r^{k-1} J_{(k-2) / 2}(r|x|)(r|x|)^{-(k-2) / 2}\right\} d r .
\end{aligned}
$$

It then follows from (4) that 


$$
H_{R}^{\alpha}(x)=c(k, \alpha) J_{k / 2+\alpha}(R|x|) R^{k / 2-\alpha}|x|^{-(k / 2+\alpha)}
$$

where

$$
\begin{aligned}
c(k, \alpha) & =2^{k / 2+\alpha-1} \Gamma(1 / 2) \Gamma(\alpha+1) \Gamma[(k-1) / 2](2 \pi)^{-k} \omega_{k-2} \\
& =2^{\alpha} \Gamma(\alpha+1) /(2 \pi)^{k / 2} .
\end{aligned}
$$

Since the inner integral in (1) was designated by $(2 \pi)^{k} H_{R}^{\alpha}(x-y)$, we have from (1) and (5) that

$$
\begin{aligned}
\tau_{R}^{\alpha}(g, x) & =\int_{E_{k}} g(y) H_{R}^{\alpha}(x-y) d y \\
& =c(k, \alpha) R^{k / 2-\alpha} \int_{E_{k}} g(y) J_{k / 2+\alpha}(R|x-y|)|x-y|^{-(k / 2+\alpha)} d y .
\end{aligned}
$$

With no loss in generality, we can take $x_{0}=0$. Setting $G(r)$ $=\int_{B(0, r)}|g(y)| d y$, we see from (6) that to prove the lemma we have to show that $G(r)=o\left(r^{k}\right)$ as $r \rightarrow 0$ implies that

(7) $\quad R^{k / 2-\alpha} \int_{0}^{\infty}\left|J_{k / 2+\alpha}(R s)\right||s|^{-(k / 2+\alpha)} d G(s)=o(1) \quad$ as $R \rightarrow \infty$.

To establish (7), we need two further facts concerning Bessel functions (see [35, p. 199]), namely that there is a constant $c_{\nu}$ such that

$$
\left|J_{\nu}(s)\right|=c_{\nu} s^{\nu} \quad \text { as } s \rightarrow 0 \text { for } \nu>-1,
$$

and that

$$
\left|J_{\nu}(s)\right| \leqq c_{\nu} s^{-1 / 2} \quad \text { as } s \rightarrow \infty \text { for } \nu \geqq-\frac{1}{2} .
$$

Using (8) and the fact that $G(s)=o\left(s^{k}\right)$ as $s \rightarrow 0$, we see that

$$
\begin{aligned}
R^{k / 2-\alpha} \int_{0}^{R-1}\left|J_{k / 2+\alpha}(R s)\right||s|^{-(k / 2+\alpha)} d G(s) & \leqq c_{k / 2+\alpha} R^{k} G\left(R^{-1}\right) \\
& =o(1) \text { as } R \rightarrow \infty .
\end{aligned}
$$

Using (9), the fact that $g$ is in $L^{1}$ on $E_{k}$, and also that $\alpha>(k-1) / 2$, we see that for every $\delta>0$,

$$
\begin{aligned}
R^{k / 2-\alpha} \int_{\delta}^{\infty} & \left|J_{k / 2+\alpha}(R s)\right||s|^{-(k / 2+\alpha)} d G(s) \\
& \leqq c_{k / 2+\alpha} R^{-[\alpha-(k-1) / 2]} \int_{E_{k}-B(0,8)}|g(y)| /|y|^{\alpha+(k+1) / 2} d y \\
& =o(1) \text { as } R \rightarrow \infty
\end{aligned}
$$


Finally using (9) once again, we see that

$$
\begin{aligned}
R^{k / 2-\alpha} \int_{R^{-1}}^{\delta}\left|J_{k / 2+\alpha}(R s)\right||s|^{-(k / 2+\alpha)} d G(s) & \\
& \leqq c_{k / 2+\alpha} R^{-[\alpha-(k-1) / 2]} \int_{R^{-1}}^{\delta} d G(s) /|s|^{[\alpha+(k+1) / 2]} .
\end{aligned}
$$

From (10), (11), and (12) we see that for every $\delta>0$,

$$
\begin{aligned}
\limsup _{R \rightarrow \infty} R^{k / 2-\alpha} \int_{0}^{\infty}\left|J_{k / 2+\alpha}(R s)\right||s|^{-(k / 2+\alpha)} d G(s) \\
\leqq c_{k / 2+\alpha} \limsup _{R \rightarrow \infty} R^{-[\alpha-(k-1) / 2]} \int_{R^{-1}}^{\delta} d G(s) /|s|^{[\alpha+(k+1) / 2]} .
\end{aligned}
$$

But $G(s)=o\left(s^{k}\right)$ as $s \rightarrow 0$ implies that the right side of (13) goes to zero as $\delta \rightarrow 0$, as an integration by parts shows. Consequently ( 7 ) is established, and therefore the lemma is established.

Lemma 2. Let $S(x)$ be the trigonometric polynomial $\sum_{|m| \leqslant R_{1}} b_{m} e^{i(m, x)}$, i.e. $S(x)=\sum_{m} b_{m} e^{i(m, x)}$ where $b_{m}=0$ for $|m|>R_{1}$. For $R>0$, set $\sigma_{R}^{\alpha}(S, x)=\sum_{|m| \leq R} b_{m} e^{i(m, x)}\left(1-|m|^{2} / R^{2}\right)^{\alpha}$. Then for $\alpha>(k-1) / 2$,

$$
\sigma_{R}^{\alpha}(S, x)=c(k, \alpha) R^{k / 2-\alpha} \int_{E_{k}} S(y) J_{k / 2+\alpha}(R|x-y|)|x-y|^{-(k / 2+\alpha)} d y
$$

where $c(k, \alpha)$ is the constant in (5).

To prove Lemma 2 , set $\phi(t)=\left(1-t^{2}\right)^{\alpha}, 0 \leqq t \leqq 1$, and $\phi(t)=0$ for $t \geqq 1$. Then since $S(x)$ is a finite linear combination of exponentials, it is clear that the lemma will follow if we can show that for fixed $x$ and every $u$

$$
\begin{aligned}
& e^{i(u, x)} \phi(|u| / R) \\
& \quad=c(k, \alpha) R^{k / 2-\alpha} \int_{E_{k}} e^{i(u, v)} J_{k / 2+\alpha}(R|x-y|)|x-y|^{-(k / 2+\alpha)} d y .
\end{aligned}
$$

Set $g(u)=e^{i(u, x)} \phi(|u| / R)$. Then $g(u)$ is a continuous function which is furthermore in $L^{1}$ on $E_{k}$. If $\hat{g}(y)$ is also in $L^{1}$ on $E_{k}$, it follows from Lemma 1 and the Lebesgue dominated convergence theorem that $g(u)=\int_{E_{k}} e^{i(y, u)} \hat{g}(y) d y$. By (8) and (9), $J_{k / 2+\alpha}(R|x-y|)|x-y|-(k / 2+\alpha)$ is in $L^{1}$ on $E_{k}$. Therefore to establish Lemma 2 we need only show that

$$
\hat{g}(y)=c(k, \alpha) R^{k / 2-\alpha} J_{k / 2+\alpha}(R|x-y|)|x-y|^{-(k / 2+\alpha)} .
$$


But $\hat{g}(y)=H_{R}^{\alpha}(x-y)$ and this last fact follows from (5). Lemma 2 is therefore established.

Lemma 3. Let $f(x)$ be in $L^{1}$ on $T_{k}$ and extended by periodicity to all of $E_{k}$. Then there exists a sequence of trigonometric polynomials $\left\{S^{n}(x)\right\}_{n=1}^{\infty}$ such that $\int_{T_{k}}\left|S^{n}(x)-f(x)\right| d x \rightarrow 0$ as $n \rightarrow \infty$.

Set

$$
K_{n}(t)=\sum_{j=-n}^{n} e^{i j t}(1-|j| / n),
$$

(so that $K_{n}(t)$ is the well-known Fejer kernel), and set $S^{n}(x)$ $=(2 \pi)^{-k} \int_{T_{k}} K_{n}\left(y_{1}\right) \cdots K_{n}\left(y_{k}\right) f(y+x) d y$. Then, for $0<\delta<\pi$,

$$
\begin{aligned}
\int_{T_{k}}\left|S^{n}(x)-f(x)\right| d x \leqq & \int_{-\delta}^{\delta} \cdots \int_{-\delta}^{\delta} K_{n}\left(y_{1}\right) \cdots K_{n}\left(y_{k}\right) d y \\
& \cdot \int_{T_{k}}|f(x+y)-f(x)| d x+o(1)
\end{aligned}
$$

as $n \rightarrow \infty$, and Lemma 3 is established.

We are now ready to prove Theorem 1 . We first observe from (5), (8), and (9) that there is a constant $N(\alpha, R)$ and an $\eta>0$ such that for $x$ in $E_{k},\left|H_{R}^{\alpha}(x)\right| \leqq N(\alpha, R)[1+|x|]^{-(k+\eta)}$. Consequently, the series

$$
\sum_{m} H_{R}^{\alpha}(x+2 \pi m)=H_{R}^{* \alpha}(x)
$$

is absolutely convergent, and furthermore

$$
\lim _{R_{1} \rightarrow \infty} \sum_{|m| \leqq R_{1}} H_{R}^{\alpha}(x+2 \pi m)=H_{R}^{* \alpha}(x)
$$

uniformly for $x$ in a bounded domain.

Let $\left\{S^{i}(x)\right\}_{j+1}^{\infty}$ be the sequence of trigonometric polynomials given by Lemma 3 . Then by Lemma 2 for $x$ in a bounded domain,

$$
\begin{aligned}
\sigma_{R}^{\alpha}\left(S^{j}, x\right) & =\int_{E_{k}} S^{j}(y) H_{R}^{\alpha}(x-y) d y \\
& =\lim _{R_{1} \rightarrow \infty} \sum_{|m| \leqq R_{1}} \int_{T_{k}} S^{j}(y+2 \pi m) H_{R}^{\alpha}(x-y-2 \pi m) d y \\
& =\lim _{R_{1} \rightarrow \infty} \sum_{|m| \leqq R_{1}} \int_{T_{k}} S^{j}(y) H_{R}^{\alpha}(y-x+2 \pi m) d y \\
& =\int_{T_{k}} S^{j}(y) H_{R}^{* \alpha}(y-x) d y .
\end{aligned}
$$


Consequently since $H_{R}^{* \alpha}(x)$ is a continuous periodic function and since $\sigma_{R}^{\alpha}\left(S^{i}, x\right) \rightarrow \sigma_{R}^{\alpha}(f, x)$ as $j \rightarrow \infty$, we conclude that

$$
\sigma_{R}^{\alpha}(f, x)=\int_{T_{k}} f(y) H_{R}^{* \alpha}(y-x) d y .
$$

Observing that $\left.f(y)(1+|y-x|)^{-(k+\eta)}\right)$ is in $L^{1}$ on $E_{k}$ for fixed $x$ since $\int_{B\left(0, R_{1}+1\right)-B\left(0, R_{1}\right)}|f(y)| d y=O\left(R_{1}^{k-1}\right)$ as $R_{1} \rightarrow \infty$, we see that we can reverse the above argument and obtain that

$$
\sigma_{R}^{\alpha}(f, x)=\int_{E_{\boldsymbol{k}}} f(y) H_{R}^{\alpha}(y-x) d y .
$$

To prove the theorem with no loss in generality we can assume $x_{0}=0$ and $f\left(x_{0}\right)=0$. Then locally the same proof will apply here as applied in the proof of Lemma 1 (as can be seen easily by comparing (6) and (15)). Therefore to complete the proof of this theorem we need only show that for fixed $\delta>0$,

$$
\lim _{R \rightarrow \infty} \int_{E_{k}-B(0,8)} f(y) H_{R}^{\alpha}(y) d y=0 .
$$

But

$$
\begin{aligned}
& R^{k / 2-\alpha} \int_{E_{k}-B(0, \delta)}|f(y)| J_{k / 2+\alpha}(R|y|)|y|^{-(k / 2+\alpha)} d y \\
& \quad \leqq c_{k / 2+\alpha} R^{((k-1) / 2)-\alpha} \int_{E_{k}-B(0,8)} \frac{|f(y)|}{|y|^{\alpha+(k+1) / 2}} d y=o(1) \text { as } R \rightarrow \infty
\end{aligned}
$$

and the proof of Theorem 1 is complete.

We next prove a theorem concerning the Abel summability of multiple Fourier series which is a $k$-dimensional analogue of the wellknown theorem of Fatou. (For another analogue which is useful, see [26, p. 606].)

THEOREM 2. Let $f$ be in $L^{1}$ on $T_{k}$ and extended by periodicity to all of $E_{k}$. Let $S[f]=\sum_{m} \hat{f}(m) e^{i(m, x)}$ and for $t>0$, set

$$
A_{t}(f, x)=\sum_{m} f(m) e^{i(m, x)-|m| t} .
$$

Also set

$$
\beta^{-}(x)=\limsup _{h \rightarrow 0}|B(x, h)|^{-1} \int_{B(x, h)} f(y) d y
$$

and 


$$
\beta_{-}(x)=\liminf _{h \rightarrow 0}|B(x, h)|^{-1} \int_{B(x, h)} f(y) d y .
$$

Then

$$
\beta_{-}(x) \leqq \liminf _{t \rightarrow 0} A_{t}(f, x) \leqq \limsup _{t \rightarrow 0} A_{t}(f, x) \leqq \beta^{-}(x)
$$

To prove Theorem 2 , we proceed in a similar manner to the proof of Theorem 1. First let $g(x)$ be in $L^{1}$ an $E_{k}$. Then for $t>0$,

$$
\int_{E_{k}} \hat{g}(y) e^{i(y, x)-|y| t} d y=(2 \pi)^{-k} \int_{E_{k}} g(u)\left[\int_{E_{k}} e^{i(x-u, y)-|y| t} d y\right] d u .
$$

By (2) and (4)

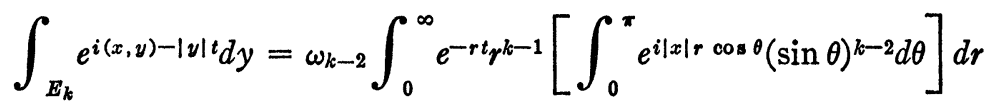

$$
\begin{aligned}
& =2^{(k-2) / 2} \Gamma[(k-1) / 2] \Gamma\left(\frac{1}{2}\right) \omega_{k-2} \\
& \cdot \int_{0}^{\infty} e^{-r t} r^{k-1} J_{(k-2) / 2}(r|x|)(r|x|)^{-(k-2) / 2} d r .
\end{aligned}
$$

Now, as easily seen, the following formula $[35$, p. 386] holds:

$$
\int_{0}^{\infty} e^{-a r} J_{\nu}(b r) r^{\nu+1} d r=\frac{2 a(2 b)^{\nu} \Gamma(\nu+3 / 2)}{\left(a^{2}+b^{2}\right)^{\nu+3 / 2} \sqrt{ } \pi} \quad \text { for } \nu>-1 .
$$

Setting $b_{k}=2^{k-1} \Gamma[(k-1) / 2] \Gamma[(k+1) / 2] \omega_{k-2}(2 \pi)^{-k}$, we conclude that for $t>0$,

$$
\int_{E_{k}} \hat{g}(y) e^{i(y, x)-|y| t} d y=b_{k} t \int_{E_{k}} g(u)\left[t^{2}+|x-u|^{2}\right]^{-(k+1) / 2} d u .
$$

Using the same techniques to pass from Fourier integrals to Fourier series that we used in the proof of Theorem 1, we consequently obtain that

$$
A_{t}(f, x)=b_{k} t \int_{E_{k}} f(u)\left[t^{2}+|x-u|^{2}\right]^{-(k+1) / 2} d u .
$$

To prove Theorem 2 , we see first that it is sufficient to establish $\lim \sup _{t \rightarrow 0} A_{t}(f, x) \leqq \beta^{-}(x)$. Next, we observe that we need only establish this last fact for the special case $x=0$. In other words to prove the theorem, it is sufficient to show that 


$$
\limsup _{t \rightarrow 0} A_{t}(f, 0) \leqq \beta^{-}(0) .
$$

If $\beta^{-}(0)=+\infty$, then $(18)$ is immediate. Two cases then present themselves, either $\beta^{-}(0)$ is finite or $\beta^{-}(0)=-\infty$. (18) will be established in both of these cases if we can show

$$
\text { if } \beta^{-}(0)<\gamma, \quad \text { then } \limsup _{t \rightarrow 0} A_{t}(f, 0) \leqq \gamma .
$$

We now establish (19) and consequently the theorem.

We set

$$
f_{r}(0)=|B(0, r)|^{-1} \int_{B(0, r)} f(u) d u,
$$

and by (19) choose $\delta>0$ such that for $0<r<\delta, f_{r}(0)<\gamma$. Observing that $f(u) /|u|^{k+1}$ is in $L^{1}$ on $E_{k}-B(0, \delta)$, we then obtain from (17) that

$$
\begin{aligned}
\limsup _{t \rightarrow 0} A_{t} & (f, 0) \\
& \leqq b_{k}|B(0,1)| \limsup t \int_{t \rightarrow 0}^{\delta}\left[t^{2}+r^{2}\right]^{-(k+1) / 2} d r^{k} f_{r}(0) \\
& \leqq b_{k}|B(0,1)|(k+1) \lim \sup _{t \rightarrow 0} t \int_{0}^{\delta} r^{k+1} f_{r}(0)\left[t^{2}+r^{2}\right]^{-(k+3) / 2} d r \\
& \leqq \gamma b_{k}|B(0,1)|(k+1) \int_{0}^{\infty} t^{k+1}\left[1+t^{2}\right]-(k+3) / 2 d t .
\end{aligned}
$$

But $b_{k}|B(0,1)|(k+1) \int_{0}^{\infty} t^{k+1}\left[1+t^{2}\right]^{-(k+3) / 2} d t=1$, (19) is established, and the theorem is proved.

It is clear that one also could prove results along the lines of nontangential Abel summability (see [37, p. 101]) but we will not concern ourselves with matters of this nature here.

For other versions of the theorems proved in this section see [6, Chapter 2] and [11, Chapter 4].

3. Critical index. In this section, we shall examine in detail the situation that prevails in Bochner-Riesz summability (Theorem 1) when $\alpha$ equals the critical index $(k-1) / 2$. Some rather interesting things occur. In the first place, a result is obtained for functions in $L^{1}$ on $E_{k}$ whose analogue is false for functions in $L^{1}$ on $T_{k}$ where as usual $k \geqq 2$. In the second place, the result is true in one-dimension for functions both in $L^{1}$ on $E_{1}$ and in $L^{1}$ on $T_{1}$. (The critical index for $k=1$ is $\alpha=0$ which is the same as ordinary convergence.)

Theorem $3[3, p .186]$. Let $g$ be in $L^{1}$ on $E_{k}$. Set 


$$
\tau_{R}^{(k-1) / 2}(g, x)=\int_{B(0, R)} e^{i(x, u)} \hat{g}(u)\left[1-|u|^{2} / R^{2}\right]^{(k-1) / 2} d u .
$$

Suppose there exists $a \delta>0$ such that $g(x)=0$ for $x$ in $B(0, \delta)$. Then $\lim _{R \rightarrow \infty} \tau_{R}^{(k-1) / 2}(g, 0)=0$.

By (6) we observe that

$$
\begin{aligned}
& \tau_{R}^{(k-1) / 2}(g, 0) \\
& \quad=c[k,(k-1) / 2] R^{1 / 2} \int_{E_{k}-B(0,8)} g(y) J_{k-1 / 2}(R|y|)|y|^{-(k-1 / 2)} d y .
\end{aligned}
$$

On the other hand, by $\left[35\right.$, p. 199], there exists a constant $d_{\nu}$ such that for $r>0$ and $\nu \geqq 1 / 2$,

$$
\left|J_{\nu}(r)-2^{1 / 2}(r \pi)^{-1 / 2} \cos [r-\pi / 4-\nu \pi / 2]\right| \leqq d_{\nu} r^{-3 / 2} .
$$

We consequently conclude from (21) and (22) that to establish Theorem 3, we need only show that

$$
\lim _{R \rightarrow \infty} \int_{E_{k}-B(0, \delta)} g(y) \cos [R|y|-k \pi / 2]|y|^{-k} d y=0 .
$$

But (23) follows immediately from the Riemann-Lebesgue lemma for functions in $L^{1}$ on $E_{1}$ and the proof of Theorem 3 is complete.

We next establish the ingenious counter-example of Bochner [3, p. 193] showing that the analogue of Theorem 3 is false for functions in $L^{1}$ on $T_{k}$.

THEOREM 4. Given $1>\delta>0$, there exists a function $f$ in $L^{1}$ on $T_{k}$ such that $f(x)=0$ in $B(0, \delta)$ and such that

$$
\limsup _{R \rightarrow \infty}\left|\sigma_{R}^{(k-1) / 2}(f, 0)\right|=\infty,
$$

where $\sigma_{R}^{(k-1) / 2}(f, x)=\sum_{|m| \leq R} \hat{f}(m) e^{i(m, x)}\left(1-|m|^{2} / R^{2}\right)^{(k-1) / 2}$.

To prove Theorem 4, we set

$$
R^{\beta} \sum_{|m| \leq R} e^{i(m, x)}\left(1-|m|^{2} / R^{2}\right)^{((k-1) / 2)+\beta}=\Phi_{R}^{\beta}(x), \quad \beta \geqq 0,
$$

and shall write $\Phi_{R}^{0}(x)$ as $\Phi_{R}(x)$. Then it is easy to show using the standard techniques that Theorem 4 will follow once we establish the following:

there exists an $x_{0}$ in $T_{k}-B(0, \delta)$ and an increasing sequence 


$$
\left\{R_{j}\right\}_{j=1}^{\infty} \text { such that } \lim _{j \rightarrow \infty}\left|\Phi_{R_{j}}\left(x_{0}\right)\right|=\infty \text {. }
$$

For consider the Banach space $B$ consisting of all real-valued functions in $L^{1}$ on $T_{k}$ which vanish almost everywhere in $B(0, \delta)$. Then $\sigma_{R_{j}}^{(k-1) / 2}(f, 0)=(2 \pi)^{-k} \int_{T_{k}} f(x) \Phi_{R j}(x) d x$ gives rise to a sequence of bounded linear functionals $F_{j}$ on $\leftrightarrow$, i.e. $F_{j}(f)=\sigma_{R_{j}}^{(k-1) / 2}(f, 0)$. If the conclusion to Theorem 4 is false, then $\sup _{j}\left|F_{j}(f)\right|$ is finite for each $f$ in B. But then by the Banach-Steinhaus theorem, $\sup _{j}\left\|F_{j}\right\|$ is finite. However

$$
\left\|F_{j}\right\|=(2 \pi)^{-k} \sup _{x \text { in } T_{k}-B(0, \delta)}\left|\Phi_{R_{j}}(x)\right| .
$$

Therefore $\left\|F_{j}\right\| \geqq(2 \pi)^{-k}\left|\Phi_{R_{j}}\left(x_{0}\right)\right|$ with $x_{0}$ given by (25), and consequently $\sup _{j}\left|\Phi_{R_{j}}\left(x_{0}\right)\right|$ is finite. But this is a contradiction to (25); and we conclude that Theorem 4 is valid.

Therefore to establish Theorem 4, we only need to establish (25). We shall show even more (see Lemma 6 and the paragraph preceding it), namely $\lim \sup _{R \rightarrow \infty}\left|\Phi_{R}(x)\right|=\infty$ except possibly for a set of measure zero in $T_{k}$. In order to do this we need a sequence of lemmas the first of which is the following:

LemMA 4. Let $f(t)$ be a real bounded measurable function in $0<t<\infty$ for which

$$
a(s)=\lim _{T \rightarrow \infty} T^{-1} \int_{0}^{T} f(t) e^{-i s t} d t
$$

exists for every $s$ in $0 \leqq s<\infty$. Then $a(s)$ is different from zero for at most a countable set of numberss which we shall denote by $S=\left\{s_{1}, s_{2}, \cdots\right\}$. If the numbers in $S$ are linearly independent with respect to integer coefficients, then

$$
\left|a\left(s_{1}\right)\right|+\left|a\left(s_{2}\right)\right|+\cdots<\infty \text {. }
$$

(We say the numbers in $S$ are linearly independent with respect to integer coefficients if $\left(c_{1}, \cdots, c_{n}\right)$ is a set of integers with $c_{1}^{2}+\cdots$ $+c_{n}^{2} \neq 0$, then $\sum_{j=1}^{n} c_{j} s_{j} \neq 0$.)

We first note that indeed $a(s) \neq 0$ for only a countable set of nonnegative real numbers. For let $r_{1}, \cdots, r_{n}$ be a set of distinct nonnegative real numbers, then

$$
T^{\sim 1} \int_{0}^{T}\left[f(t)-\sum_{j=1}^{n} a\left(r_{j}\right) e^{i r_{j} t}\right]\left[f(t)-\sum_{j=1}^{n} \bar{a}\left(r_{j}\right) e^{-i r_{j} t}\right] d t \geqq 0 .
$$

Letting $M$ be the $L^{\infty}$-norm of $f$ on $(0, \infty)$ and observing that for 
$\beta \neq 0, T^{-1} \int_{0}^{T} e^{i \beta t} d t \rightarrow 0$ as $T \rightarrow \infty$, we see from (26) that $\sum_{j=1}^{n}\left|a\left(r_{j}\right)\right|^{2}$ $\leqq M^{2}$; consequently the set $S$ of the lemma is at most countable.

(Note that zero is not in $S$ because of linear independence.)

Next for $s_{j}$ in $S$, we write $a\left(s_{j}\right)=\left|a\left(s_{j}\right)\right| e^{-i \beta_{j}}$ and form

$$
K_{q}(t)=\prod_{j=1}^{q} K\left(s_{j} t-\beta_{j}\right) \geqq 0
$$

where $K(t)=1+\left(e^{i t}+e^{-i t}\right) 2^{-1}=1+\cos t \geqq 0$.

Then from (27) we observe that

$$
K_{q}(t)=1+2^{-1} \sum_{j=1}^{q}\left[e^{i \delta_{j} t} e^{-i \beta_{j}}+e^{-i \delta_{j} t} e^{i \beta_{j}}\right]+R_{q}(t)
$$

where $R_{q}(t)$ is a finite linear combination of $e^{i\left(e_{1} s_{1}+\cdots+e_{q} s_{q}\right) t}$ with $\epsilon_{p}=0$ or \pm 1 and at least two $\epsilon_{p}$ are different from zero. Consequently, because of linear independence, $\epsilon_{1} s_{1}+\cdots+\epsilon_{q} s_{q}$ is not in $S$ and not equal to zero. Likewise $-\left(\epsilon_{1} s_{1}+\cdots+\epsilon_{q} s_{q}\right)$ is not in $S$ and also is not equal to zero. Therefore

$$
\lim _{T \rightarrow \infty} T^{-1} \int_{0}^{T} f(t) R_{q}(t) d t=0 \text { and } \lim _{T \rightarrow \infty} T^{-1} \int_{0}^{T} R_{q}(t) d t=0 .
$$

We conclude from (28) and (29) that

$$
\begin{aligned}
\lim _{T \rightarrow \infty} T^{-1} \int_{0}^{T} f(t) K_{q}(t) d t & =2^{-1} \sum_{j=1}^{q}\left[\bar{a}\left(s_{j}\right) e^{-i \beta_{j}}+a\left(s_{j}\right) e^{i \beta_{j}}\right] \\
& =\sum_{j=1}^{q}\left|a\left(s_{j}\right)\right| .
\end{aligned}
$$

On the other hand by (27), (28), and (29)

$$
\lim _{T \rightarrow \infty} T^{-1} \int_{0}^{T} f(t) K_{q}(t) d t \leqq M \lim _{T \rightarrow \infty} T^{-1} \int_{0}^{T} K_{q}(t) d t=M .
$$

We conclude that

$$
\sum_{j=1}^{q}\left|a\left(s_{j}\right)\right| \leqq M
$$

and Lemma 4 is established.

From Lemma 4, we see that the first step in establishing (25) is to show that $R^{-1} \int_{0}^{R} \Phi_{r}(x) e^{-i \lambda r} d r$ tends to a finite limit for $0 \leqq \lambda<\infty$. We do this with the following lemma:

Lemma 5. If $x$ is not of the form $2 \pi m$ where $m$ is a lattice point, then for each $\lambda$ in $0 \leqq \lambda<\infty$ 


$$
\lim _{R \rightarrow \infty} R^{-1} \int_{0}^{R} \Phi_{r}(x) e^{-i \lambda r} d r
$$

exists. It equals 0 if $\lambda \neq \lambda_{m}(x)$ for every $m$, and it equals $c_{k}\left|\lambda_{m}(x)\right|^{-k}$ if $\lambda=\lambda_{m}(x)$ where

$$
\lambda_{m}(x)=|2 \pi m-x|
$$

and $c_{k}$ is a nonzero constant.

To establish Lemma 5, we first establish the following remark $[31$, p. 91$]$.

REMARK 1. Let $g$ be a continuous function in $L^{1}$ on $E_{k}$ and let $\hat{g}$ be its Fourier transform. Suppose there exists a constant $A$ and an $\epsilon>0$ such that $|g(x)| \leqq A(1+|x|)^{-(k+\epsilon)}$ and $|\hat{g}(x)| \leqq A(1+|x|)^{-(k+\epsilon)}$. Then

$$
\sum_{m} g(x-2 \pi m)=\sum_{m} \hat{g}(m) e^{i(m, x)} .
$$

To prove the above remark, we note that both sides of (30) converge absolutely uniformly for $x$ in any bounded domain and represent periodic functions of period $2 \pi$ in each variable. Furthermore, the $m_{0}$ th Fourier coefficient of the right side of $(30)$ is $\hat{g}\left(m_{0}\right)$. The $m_{0}$ th Fourier coefficient of the left side of (27) is

$$
(2 \pi)^{-k} \int_{T_{k}} e^{-i\left(m_{0}, x\right)} \sum_{m} g(x-2 \pi m) d x=(2 \pi)^{-k} \int_{E_{k}} g(x) e^{-i\left(m_{0}, x\right)} d x=\hat{g}\left(m_{0}\right)
$$

and the remark is established.

Taking $g(x)$ in Remark 1 as $J_{k+\beta-1 / 2}(R|x|) /|x|^{k+\beta-1 / 2}, \beta>0$, we observe from (14) with $\alpha=(k-1) / 2+\beta$ and $c(k, \alpha)=2^{\alpha} \Gamma(\alpha+1) /(2 \pi)^{k / 2}$ that

$$
\begin{aligned}
c(k,(k-1) / 2+\beta) R^{1 / 2-\beta} \int_{E_{k}} e^{-i(y, x)} \frac{J_{k+\beta-1 / 2}(R|x|)}{|x|^{k+\beta-1 / 2}} d x & \\
=\left(1-|y|^{2} / R^{2}\right)^{(k-1) / 2+\beta} & \text { for }|y| \leqq R \\
=0 & \text { for }|y|>R .
\end{aligned}
$$

We conclude from Remark 1 and (24) with

$$
b(k, \beta)=c(k,(k-1) / 2+\beta)(2 \pi)^{k+1 / 2}
$$

that

$$
\Phi_{R}^{\beta}(x)=\frac{b(k, \beta) R^{1 / 2}}{(2 \pi)^{1 / 2}} \sum_{m} \frac{J_{k+\beta-1 / 2}\left[R \lambda_{m}(x)\right]}{\left[\lambda_{m}(x)\right]^{k+\beta-1 / 2}}
$$


We next obtain from [35, p. 197] that there exists a constant $A(k)$ such that for $0<\beta<1 / 4$ and $t>0$,

$$
\left|J_{k+\beta-1 / 2}(t)-(2 / \pi t)^{1 / 2} \cos [t-(k+\beta) \pi / 2]\right|<A(k) / t .
$$

Consequently from (31), we see that for $0<\beta<1 / 4$ and $R>0$,

$$
\left|\Phi_{R}^{\beta}(x)-[b(k, \beta) / \pi] \sum_{m} \cos \frac{\left[R \lambda_{m}(x)-(k+\beta) \pi / 2\right]}{\left[\lambda_{m}(x)\right]^{k+\beta}}\right|<B(k) / R^{1 / 2}
$$

where $A(k)$ and $B(k)$ are constants depending on $k$ but not on $\beta$. (Of course, $B(k)$ also depends on $x$, which is fixed and not of the form $2 \pi m$.)

Therefore for $R>0$ and $1 / 4>\beta>0$,

$$
\mid R^{-1} \int_{0}^{R} \Phi_{r}^{\beta}(x) e^{-i r \lambda} d r-b(k, \beta)(2 \pi R)^{-1} \sum_{m}\left\{e^{-i(k+\beta) \pi / 2} \int_{0}^{R} e^{i r\left[\lambda_{m}(x)-\lambda\right]} d r\right.
$$

$$
\left.+e^{i(k+\beta) \pi / 2} \int_{0}^{R} e^{-i r\left[\lambda_{m}(x)+\lambda\right]} d r\right\} /\left[\lambda_{m}(x)\right]^{k+\beta} \mid<\frac{2 B(k)}{R^{1 / 2}} .
$$

If $\lambda \neq \lambda_{m}(x)$ for every lattice point $m$, we see from (32) that

$$
\begin{aligned}
\mid \frac{1}{R} \int_{0}^{R} \Phi_{r}^{\beta}(x) e^{-i r \lambda} d r & \\
& -\frac{b(k, \beta)}{2 \pi R} \sum_{m} \frac{e^{-i(k+\beta) \pi / 2}\left[e^{i\left(\lambda_{m}(x)-\lambda\right) R}-1\right]}{i\left[\lambda_{m}(x)-\lambda\right]\left[\lambda_{m}(x)\right]^{k+\beta}} \\
& +\frac{b(k, \beta)}{2 \pi R} \sum_{m} \frac{e^{i(k+\beta) / 2}\left[e^{-i\left(\lambda_{m}(x)+\lambda\right) R}-1\right]}{i\left[\lambda_{m}(x)+\lambda\right]\left[\lambda_{m}(x)\right]^{k+\beta}} \mid<\frac{2 B(k)}{R^{1 / 2}} .
\end{aligned}
$$

Passing to the limit as $\beta \rightarrow 0$, we see that for $R>0$,

$$
\begin{aligned}
\mid R^{-1} \int_{0}^{R} \Phi_{r}(x) e^{-i r \lambda} d r & \\
& -\frac{b(k, 0)}{2 \pi R} \sum_{m} e^{-i k \pi / 2} \frac{\left[e^{i\left[\lambda_{m}(x)-\lambda\right] R}-1\right]}{i\left[\lambda_{m}(x)-\lambda\right]\left[\lambda_{m}(x)\right]^{k}} \\
& +\frac{b(k, 0)}{2 \pi R} \sum_{m} e^{i k \pi / 2} \frac{\left[e^{-i\left[\lambda_{m}(x)+\lambda\right] R}-1\right]}{i\left[\lambda_{m}(x)+\lambda\right]\left[\lambda_{m}(x)\right]^{k}} \mid \leqq \frac{2 B(k)}{R^{1 / 2}} .
\end{aligned}
$$

Consequently if $\lambda \neq \lambda_{m}(x)$ for every $m$,

$$
\lim _{R \rightarrow \infty} R^{-1} \int_{0}^{R} \Phi_{r}(x) e^{-i r \lambda} d r=0 .
$$


On the other hand proceeding as above we see from (32) that if $\lambda=\lambda_{m_{0}}(x)$,

$$
\lim _{R \rightarrow \infty} R^{-1} \int_{0}^{R} \Phi_{r}(x) e^{-i \lambda r} d r=\frac{b(k, 0) e^{-i k \pi / 2}}{2 \pi\left[\lambda_{m_{0}}(x)\right]^{k}} .
$$

Since

$$
\begin{aligned}
\frac{b(k, 0)}{2 \pi} & =(2 \pi)^{k-1 / 2} c(k,(k-1) / 2) \\
& =\frac{(2 \pi)^{k-1 / 2}}{(2 \pi)^{k / 2}} 2^{(k-1) / 2} \Gamma(k+1 / 2),
\end{aligned}
$$

we see that the lemma is established.

Since every $x$ in $T_{k}-0$ is not of the form $2 \pi m$ and since furthermore $\sum_{m}\left|\lambda_{m}(x)\right|^{-k}=+\infty$, we see from Lemmas 4 and 5 that we cannot simultaneously have $\sup _{0<R<\infty}\left|\Phi_{R}(x)\right|<\infty$ and $\left\{\lambda_{m}(x)\right\}_{m}$ linearly independent with respect to integer coefficients. Consequently to establish (25) and therefore the theorem, it is sufficient to establish the following lemma ([3, p. 190] and [30, p. 166]):

Lemma 6. The sequence $\left\{\lambda_{m}(x)\right\}_{m}$ is linearly independent with respect to integer coefficients for almost every $x$ in $T_{k}$.

Let $\left\{m^{i}\right\}_{j=1}^{\infty}$ be an enumeration of the lattice points in $E_{k}$. We first observe that $\lambda_{m i}(x)$ is a real-analytic function of $x$ in $E_{k}-2 \pi m^{i}$, and consequently for integers $c_{1}, \cdots, c_{n}$ with $c_{1}^{2}+\cdots+c_{n}^{2} \neq 0$, $\sum_{j=1}^{n} c_{j} \lambda_{m i}(x)$ is a real-analytic function in $E_{k}$ except at the distinct points $2 \pi m^{1}, \cdots, 2 \pi m^{n}$. Let $A_{c_{1} \cdots c_{n}}$ be the set of points $x$ in $E_{k}$ such that $\sum_{j=1}^{n} c_{j} \lambda_{m i}(x)=0$. If $A_{c_{1} \cdots o_{n}}$ is a set of positive measure, then it is not difficult to show that

$$
\sum_{j=1}^{n} c_{j} \lambda_{m^{j}}(x)=0 \quad \text { for all } x \text { in } E_{k} .
$$

But (33) is impossible if $c_{1}^{2}+\cdots+c_{n}^{2} \neq 0$. For suppose $c_{j_{1}} \neq 0$. Then (33) implies that $\partial \lambda_{m^{i 1}}(x) / \partial x_{1}$ tends to a finite value as $x \rightarrow 2 \pi m^{i 1}$. On the other hand an easy computation shows that

$$
\lim _{x \rightarrow 2 \pi m^{j 1}} \partial \lambda_{m} j_{1}(x) / \partial x_{1}
$$

does not exist. We conclude that $A_{c_{1} \cdots c_{n}}$ is of measure zero in $E_{k}$.

Let $A$ designate the (countable) union of all such $A_{c_{1}} \ldots c_{n}$. Then $A$ is of measure zero in $E_{k}$. If $x$ is not in $A,\left\{\lambda_{m i}(x)\right\}_{j=1}^{\infty}$ is linearly independent with respect to integer coefficients. The lemma, and consequently the theorem, is established. 
We close this section by stating some recent theorems of Stein concerning Bochner-Riesz summability of multiple Fourier series at the critical index $\alpha=(k-1) / 2$.

Let $C(x, r)$ be the $(k-1)$-sphere which is the boundary of the $k$-ball $B(x, r)$, and, as before, let $\omega_{k-1}$ be the $(k-1)$-volume of $C(0,1)$, the unit $(k-1)$-sphere [see the paragraph between (4) and (5)].

Setting $f^{r}(x)=\left(\omega_{k-1} r^{k-1}\right)^{-1} \int_{c(x, r)} f(y) d c(y)$ where $d c(y)$ is the natural $(k-1)$-volume element on $C(x, r)$, and setting $\delta(x, r)=f^{r}(x)-f(x)$, we shall say $f$ satisfies a Dini condition at $x$ if there exists an $\eta>0$ such that

$$
\int_{0}^{\eta} r^{-1}|\delta(x, r)| d r<\infty
$$

The following theorem then prevails $[31$, p. 107],

Theorem 5. Let $|f| \log ^{+}|f|$ be in $L^{1}$ on $T_{k}$ and let $f$ be extended by periodicity to all of $E_{k}$. Also, let $S[f]=\sum_{m} \hat{f}(m) e^{i(m, x)}$. Suppose that $f$ satisfies a Dini condition at $x_{0}$. Then

$$
\lim _{R \rightarrow \infty} \sum_{|m| \leq R} \hat{f}(m) e^{i\left(m, x_{0}\right)}\left(1-|m|^{2} / R^{2}\right)^{(k-1) / 2}=f\left(x_{0}\right) .
$$

We note that if $f$ is in $L^{p}$ on $T_{k}$ for $p>1$, then $|f| \log ^{+}|f|$ is in $L^{1}$ on $T_{k}$ and that the case $f$ is in $L^{2}$ on $T_{k}$ is already contained in the original paper of Bochner [3, p. 207].

For the proof of the above theorem, we refer the reader to Stein's paper [31]. The essential idea in the proof is to make a nontrivial estimate of the difference of the corresponding "Dirichlet" kernels on $T_{k}$ and $E_{k}$, i.e. of the difference

$$
\sum_{|m| \leqq R} e^{i(m, x)}\left(1-|m|^{2} / R^{2}\right)^{(k-1) / 2}-\int_{B(0, R)} e^{i(x, y)}\left(1-|y|^{2} / R^{2}\right)^{(k-1) / 2} d y .
$$

Stein furthermore puts the critical index in proper perspective by establishing the following generalization of Kolmogorov's classical result on one dimensional Fourier series.

TheOREM 6. There exists an $f$ in $L^{1}$ on $T_{k}$ with $S[f]=\sum_{m} \hat{f}(m) e^{i(m, x)}$ such that for almost every $x$ in $T_{k}$,

$$
\limsup _{R \rightarrow \infty}\left|\sum_{|m| \leqq R} \hat{f}(m) e^{i(m, x)}\left(1-|m|^{2} / R^{2}\right)^{(k-1) / 2}\right|=+\infty .
$$

For the proof of the above theorem, we refer the reader to Stein's paper $[30$, p. 165]. The essential idea in the proof is to apply a theorem proved in the paper concerning weak type operators. 
4. Uniqueness. We now turn our attention to a different aspect of multiple Fourier series, namely the uniqueness theory of multiple trigonometric series.

In view of Theorem 2, one is led naturally to the following question: If $\lim _{t \rightarrow 0+} \sum_{m} a_{m} e^{i(m, x)-|m| t}=0$ for all $x$ in $T_{k}$, is $a_{m}=0$ for all $m$ ?

Since the series with coefficients $a_{m}$ defined by $a_{m}=0$ for $m_{2}^{2}+\ldots$ $+m_{k}^{2} \neq 0$ and $a_{m}=m_{1}$ for $m_{2}^{2}+\cdots+m_{k}^{2}=0$ has the property that $\lim _{t \rightarrow 0+} \sum_{m} a_{m} e^{i(m, x)-|m| t}=0$ for all $x$, we see that another condition is needed on the $a_{m}$ in order to answer the above question in the affirmative. A natural condition, in view of the above, is $\sum_{R-1<|m| \leqslant R}\left|a_{m}\right|=o(R)$ as $R \rightarrow \infty$. The following theorem established by Shapiro [25], prevails.

THEOREM 7. Given the multiple trigonometric series $\sum_{m} a_{m} e^{i(m, x)}$ where the $a_{m}$ are arbitrary complex numbers. Suppose that

(i) $\sum_{R-1<|m| \leqslant R}\left|a_{m}\right|=o(R)$ as $R \rightarrow \infty$,

(ii) $\lim _{t \rightarrow 0} \sum_{m} a_{m} e^{i(m, x)-|m| t}=0$ for $x$ in $T_{k}-0$.

Then $a_{m}=0$ for all $m$.

Before proving the above theorem, we would like to make a number of comments. In the first place, in view of the preceding remarks the condition (i) cannot be replaced by $\sum_{R-1<|m| \leq R}\left|a_{m}\right|=0(R)$ as $R \rightarrow \infty$. In the second place the above theorem is false in one-dimension (we are assuming throughout this survey that $k \geqq 2$ ) as is easily seen from a consideration of the Fourier-Stieltjes series of the unit mass placed at the origin in $T_{1}$. However, if (ii) is replaced by the condition " 0 in all of $T_{1}$," then the above theorem is true in onedimension and is due to Verblunsky. (For a history of the aspects of the above theorem, see [37, pp. 382-383] and many other places.) The $k$-dimensional version requires several new and interesting ideas.

A much more general version of Theorem 7 involving upper and lower limits of the Abel partial sums and functions in $L^{1}$ on $T_{k}$ actually prevails. For the full statement and proof of these theorems due to Shapiro, we refer the reader to [25] (and also to [27, Chapter 1] where analogous theorems for the two-sphere are established. See also $[19 ; 12 ; 17 ; 18])$.

In order to prove Theorem 7 , we first need the concept of an upper and lower generalized Laplacian which we define as follows:

Let $G(x)$ be a function in $L^{1}$ in a neighborhood of the point $x_{0}$, then using the notation previously introduced (see (20)), set $G_{h}\left(x_{0}\right)$ $=\left|B\left(x_{0}, h\right)\right|^{-1} \int_{B\left(x_{0}, h\right)} G(x) d x$. Then we call $\Delta^{*} G\left(x_{0}\right)$ the upper generalized Laplacian of $G$ at $x_{0}$ and define it by 


$$
\Delta^{*} G\left(x_{0}\right)=2(k+2) \limsup _{h \rightarrow 0}\left[G_{h}\left(x_{0}\right)-G\left(x_{0}\right)\right] / h^{2} .
$$

In a similar manner, we define $\Delta_{*}$ using $\lim \inf _{h \rightarrow 0}$.

It is to be noticed that if $G(x)$ is also in class $C^{2}$ in a neighborhood of $x_{0}$, then $\Delta^{*} G\left(x_{0}\right)=\Delta_{*} G\left(x_{0}\right)=\Delta G\left(x_{0}\right)$ where $\Delta$ designates the usual Laplace operator.

The following lemma then prevails:

Lemma 7. Let $G(x)$ be a function in $L^{1}$ on $T_{k}$ and extended by periodicity to all of $E_{k}$. Let $S[G]=\sum_{m} \hat{G}(m) e^{i(m, x)}$ and for $t>0$, set $G(x, t)$ $=\sum_{m} \hat{G}(m) e^{i(m, x)-|m| t}$. Suppose $\lim _{t \rightarrow 0+} G\left(x_{0}, t\right)=G\left(x_{0}\right)$ exists and is finite. Set $\gamma^{*}\left(x_{0}\right)=\lim \sup _{t \rightarrow 0+}-\sum_{m}|m|^{2} \hat{G}(m) e^{i\left(m, x_{0}\right)-|m| t}$ and define $\gamma_{*}\left(x_{0}\right)$ similarly using $\lim \inf _{t \rightarrow 0+}$. Then

(a) $\Delta_{*} G\left(x_{0}\right) \leqq \gamma^{*}\left(x_{0}\right)$ and

(b) $\gamma_{*}\left(x_{0}\right) \leqq \Delta^{*} G\left(x_{0}\right)$.

(For the one-dimensional analogue of the above result see [37, p. 353].)

To prove the lemma it is sufficient to prove (a), for (b) will then follow by considering $-G(x)$. With no loss in generality, we can also assume that $x_{0}=0$ and $G(0)=0$.

If $\Delta_{*} G(0)=-\infty$, or if $\gamma^{*}(0)=+\infty$ (a) is already established, so we can also assume that $\Delta_{*} G(0)>-\infty$ and $\gamma^{*}(0)<+\infty$.

Suppose (a) does not hold. Then there exists a constant $\eta$ such that $\Delta_{*} G(0)>\eta>\gamma^{*}(0)$. Since we can find a periodic function $\lambda(x)$ which is in class $C^{\infty}$ with the properties that $\lambda(0)=0$ and $\Delta \lambda(0)=\eta$, we can assume that $\eta=0$. We prove the lemma by showing that $\Delta_{*} G(0)$ $>0>\gamma^{*}(0)$ leads to a contradiction.

First suppose $\gamma^{*}(0)<0$. Then with $G_{t}(0, t)$ designating $d G(0, t) / d t$ we observe that $\lim \sup _{t \rightarrow 0}-G_{t t}(0, t)=\gamma^{*}(0)<0$. Consequently, $G_{t t}(0, t)>0$ for $t$ sufficiently small, and therefore for $t$ sufficiently small $G_{t}(0, t)$ is an increasing function of $t$, i.e. there exists a $t_{0}>0$ such that $G_{t}(0, t)$ is an increasing function in the interval $0<t<t_{0}$. Also $G(0, t) / t=G_{t}(0, s)$ where $0<s<t$ by the mean-value theorem, since $\lim _{t \rightarrow 0} G(0, t)=0$. Therefore $\lim \sup _{t \rightarrow 0} d[G(0, t) / t] / d t<0$ is incompatible with the fact that $G_{t}(0, t)$ is an increasing function for $0<t<t_{0}$. Consequently, if we can show that

$$
\text { if } \Delta_{*} G(0)>0, \quad \text { then } \liminf _{t \rightarrow 0}-d[G(0, t) / t] / d t>0,
$$

we shall have arrived at a contradiction.

We now establish (34). Observing that $|B(0, r)| G_{r}(0)=\int_{B(0, r)} G(u) d u$ we obtain from (17) that for $t>0$, 


$$
\begin{aligned}
G(0, t) / t & =A \int_{0}^{\infty}\left[t^{2}+r^{2}\right]^{-(k+1) / 2} d r^{k} G_{r}(0) \\
& =A^{\prime} \int_{0}^{\infty} r^{k+1} G_{r}(0)\left[t^{2}+r^{2}\right]^{-(k+8) / 2} d r
\end{aligned}
$$

where $A$ and $A^{\prime}$ are positive constants. Since $G_{r}(0)=O(1)$ as $r \rightarrow \infty$, we conclude that

$$
d[-G(0, t) / t] / d t=A^{\prime \prime} t \int_{0}^{\infty} r^{k+1} G_{r}(0)\left[t^{2}+r^{2}\right]^{-(k+5) / 2} d t
$$

where $A^{\prime \prime}$ is a positive constant. By the assumption in (34), there exists $h_{0}>0$ such that for $0<r<\delta, G_{r}(0)>r^{2} h_{0}$. We thus obtain from (35) that

$$
\begin{aligned}
\liminf _{t \rightarrow 0} d[-G(0, t) / t] / d t & \geqq A^{\prime \prime} \liminf _{t \rightarrow 0} t \int_{0}^{\delta} r^{k+3} h_{0}\left[t^{2}+r^{2}\right]-(k+5) / 2 d r . \\
& \geqq h_{0} A^{\prime \prime} \int_{0}^{\infty} r^{k+3}\left[1+r^{2}\right]^{-(k+5) / 2} d r \\
& >0 ;
\end{aligned}
$$

(34) is established, and the proof of the lemma is complete.

We now prove the theorem. From a consideration of the series $\sum_{m}\left(a_{m}+\bar{a}_{-m}\right) e^{i(m, x)}$ and $\sum_{m} i\left(a_{m}-\bar{a}_{-m}\right) e^{i(m, x)}$, we see from the start that it is sufficient to prove the theorem under the additional assumption $\bar{a}_{m}=a_{-m}$, which we shall henceforth make.

We next set for $t>0$,

$$
\begin{aligned}
f(x, t) & =\sum_{m} a_{m} e^{i(m, x)-|m| t}, \\
f_{1}(x, t) & =-\sum_{m \neq 0} a_{m}|m|^{-1} e^{i(m, x)-|m| t}, \\
F(x, t) & =-\sum_{m \neq 0} a_{m}|m|^{-2} e^{i(m, x)-|m| t}, \\
f^{*}(x) & =\limsup _{i \rightarrow 0} f(x, t) \text { and } f_{*}(x)=\liminf _{t \rightarrow 0} f(x, t),
\end{aligned}
$$

and observe that

(37) for $x \operatorname{in} T_{k}-0, \quad \lim _{t \rightarrow 0} f_{1}(x, t)$ and $\lim _{t \rightarrow 0} F(x, t)$ exist and are finite.

To see this, fix $x$ in $T_{k}-0$. Then by (ii), there exists a constant $K$ depending on $x$ such that $|f(x, t)| \leqq K$ for $t>0$. Then by the mean-value theorem for $0<t_{1}<t_{2}$, there exists an $s$ such that 
$\left|f_{1}\left(x, t_{2}\right)-f_{1}\left(x, t_{1}\right)\right|=\left|f(x, s)-a_{0}\right|\left(t_{2}-t_{1}\right)$ where $0<t_{1}<s<t_{2}$. Therefore, $f_{1}(x, t)$ satisfies the Cauchy criterion for convergence and the first part of (37) is established. Repeating this same argument for $F(x, t)$ establishes the second part.

Using (37), we define the periodic function $F(x)$ in $E_{k}$ for $x \neq 2 \pi m$ by $F(x)=\lim _{t \rightarrow 0} F(x, t)$. As is easily seen, (i) implies that $\sum_{m \neq 0}\left|a_{m}\right|^{2} /|m|^{4}<\infty$. Consequently $F(x)$ is in $L^{2}$ on $T_{k}$ and $S[F]$ $=-\sum_{m \neq 0} a_{m} e^{i(m, x)} /|m|^{2}$. Using the notation of (20), (i), and the fact that $|B(x, h)|^{-1} \int_{B(x, h)} e^{i(m, y)} d y=\mu_{k} e^{i(m, x)} J_{k / 2}(|m| h)(|m| h)^{-k / 2}$ where $\mu_{k}$ is a constant depending on $k$ but not on $m$, we conclude that for $h>0$,

$$
F_{h}(x)=-\mu_{k} \sum_{m \neq 0} a_{m} J_{k / 2}(|m| h) e^{i(m, x)} /|m|^{2}(|m| h)^{k / 2} .
$$

(Note that it is clear from (38), that $\mu_{k}^{-1}=\lim _{t \rightarrow 0} J_{k / 2}(t) t^{-k / 2}$. Also, note that the series in (38) is absolutely convergent.)

We next obtain from (ii), (36) and Lemma 7 that

$$
\Delta^{*} F(x) \geqq-a_{0} \text { and } \Delta_{*} F(x) \leqq-a_{0} \quad \text { for } x \neq 2 \pi m .
$$

Consequently, it follows from well-known theorems concerning generalized Laplacians [21, p. 14] that if $B\left(x_{0}, h_{0}\right)$ contains no point of the form $2 \pi m$ and if $F(x)$ is continuous in $B\left(x_{0}, h_{0}\right)$, then (39) implies that $F(x)+a_{0}|x|^{2} / 2 k$ is actually harmonic in $B\left(x_{0}, h_{0}\right)$.

We now show that given such a $B\left(x_{0}, h_{0}\right), F(x)$ is actually continuous there, which is the heart of the whole proof. In order to accomplish this, we first need to establish some more facts concerning $F(x)$.

We set $\alpha_{1}(t)=\sup _{0<h \leq t} \sup _{x}$ in $T_{k}\left|F_{h}(x)-F(x, h)\right|$ and shall show that

$$
\lim _{t \rightarrow 0} \alpha_{1}(t)=0 .
$$

It follows from the definition of $F(x, h)$ in (36) and $F_{h}(x)$ in (38) that

$$
\begin{aligned}
\sup _{x \text { in } T_{k}} \mid & F_{h}(x)-F(x, h) \mid \\
& \leqq \sum_{m \neq 0}\left|a_{m}\right|\left|e^{-|m| h}-\mu_{k} J_{k / 2}(m|h|)(|m| h)^{-k / 2}\right||m|^{-2} .
\end{aligned}
$$

We split the sum on the right side of (41) into two parts, $A_{h}$ and $B_{h}$ with $A_{h}$ designating the sum over the lattice points $m, 1 \leqq|m|$ $\leqq h^{-1}$, and $B_{h}$ designating the sum over the lattice points $m,|m|$ $\geqq h^{-1}$. To establish (40), it is sufficient to show that $\lim _{h \rightarrow 0} A_{h}=0$ and $\lim _{h \rightarrow 0} B_{h}=0$.

Observing that there is a constant $K$ such that 


$$
\left|e^{-r}-\mu_{k} J_{k / 2}(r) r^{-k / 2}\right| \leqq K r \quad \text { for } 0 \leqq r<1 \text {, }
$$

we see from assumption (i) of the theorem and (41) that

$$
A_{h} \leqq K h \sum_{1 \leqq|m| \leqq h^{-1}}\left|a_{m}\right||m|^{-1}=h o\left(h^{-1}\right) .
$$

Therefore, $\lim _{h \rightarrow 0} A_{h}=0$.

Using the fact that there exists a constant $K$ such that $\left|J_{k / 2}(r)\right|$ $\leqq K r^{-1 / 2}$, we obtain from (41) and from assumption (i) of the theorem that for $h$ small,

$$
B_{h} \leqq o(1) \int_{h^{-1}-1}^{\infty} e^{-r h} r^{-1} d r+o\left(h^{-(k+1) / 2}\right) \int_{h^{-1}-1}^{\infty} r^{-(k+3) / 2} d r
$$

We conclude that $\lim _{h \rightarrow 0} B_{h}=0$, and, consequently, that (40) is established.

The next fact we establish concerning $F(x)$ is the following:

Set $\alpha_{2}(t)=\sup _{0<h \leq t} \sup _{|x-p| \leq h, x \text { and } p \text { in } E_{k}}\left|F_{h}(x)-F_{h}(p)\right|$. Then

$$
\lim _{t \rightarrow 0} \alpha_{2}(t)=0 \text {. }
$$

By (38),

$$
\begin{aligned}
& \sup _{|x-p| \leqq h, x \text { and } p \text { in } E_{k}}\left|F_{h}(x)-F_{h}(p)\right| \\
& \leqq \sup _{|x-p| \leq h, x \text { and } p \text { in } E_{k}} \mu_{k} \sum_{1 \leqq|m| \leqq h^{-1}} \\
& \quad \cdot\left\{\left|a_{m}\right|\left|J_{k / 2}(|m| h)\right|(|m| h)^{-k / 2}|m|^{-2} \mid e^{i(m, x)}-e^{i(m, p) \mid}\right\} \\
& \quad+2 \mu_{k} \sum_{h^{-1} \leq|m|}\left|a_{m}\right|\left|J_{k / 2}(|m| h)\right|(|m| h)^{-k / 2}|m|^{-2} \\
& =A_{h}^{\prime}+B_{h}^{\prime} .
\end{aligned}
$$

To establish (42), we have to show that both $A_{h}^{\prime}$ and $B_{h}^{\prime}$ tend to zero as $h$ goes to zero. That $\lim _{h \rightarrow 0} B_{h}^{\prime}=0$ was already shown when we established (40). For $A_{h}{ }^{\prime}$, we observe that

$$
\begin{aligned}
A_{h}^{\prime} & \leqq \mu_{k} \sum_{1 \leq|m| \leqq h^{-1}}\left|a_{m}\right|\left|J_{k / 2}(|m| h)\right|(|m| h)^{-k / 2}|m|^{-2}|m| h \\
& \leqq O(1) h \sum_{1 \leq|m| \leq h^{-1}}\left|a_{m}\right||m|^{-1} \\
& \leqq O(1) h o\left(h^{-1}\right) .
\end{aligned}
$$

Consequently, $\lim _{h \rightarrow 0} A_{h}^{\prime}=0$ and (42) is established.

Next, using the fact that $f(x, t)$ is continuous for $t>0$ and periodic in the $x$ variables, we select a sequence $t_{1}>t_{2}>\cdots>t_{n} \cdots \rightarrow 0$ such that 


$$
\sup _{x \text { in } E_{k}} \sup _{t_{n}+1 \leq t \leq t_{n}}\left|f(x, t)-f\left(x, t_{n}\right)\right| \leqq 1 .
$$

Now, let $B\left(x_{0}, h_{0}\right)$ be an open $k$-ball whose closure $\bar{B}\left(x_{0}, h_{0}\right)$ contains no points of the form $2 \pi m, m$ an integral lattice point. We propose to show that $F(x)+a_{0}|x|^{2} / 2 k$ is continuous in $B\left(x_{0}, h_{0}\right)$. By (ii) of the theorem, $\lim _{n \rightarrow \infty}\left|f\left(x, t_{n}\right)\right|=0$ for $x$ in $B\left(x_{0}, h_{0}\right)$. Consequently if $\bar{B}(y, s) \subset B\left(x_{0}, h_{0}\right), s>0$, there exists (by the Baire category theorem, see [37, p. $29(12.3 \mathrm{i})]) \bar{B}\left(y^{\prime}, s^{\prime}\right) \subset \bar{B}(y, s), s^{\prime}>0$, and a constant $K$ such that $\left|f\left(x, t_{n}\right)\right| \leqq K$ for $x$ in $\bar{B}\left(y^{\prime}, s^{\prime}\right)$ and $n=1,2, \cdots$. But, then by (44), $|f(x, t)| \leqq K+1$ for $0<t \leqq t_{1}$ and $x$ in $\bar{B}\left(y^{\prime}, s^{\prime}\right)$. Employing the same technique used in establishing (37), we obtain that $f_{1}(x, t)$ and $F(x, t)$ converge uniformly as $t \rightarrow 0$ for $x$ in $\bar{B}\left(y^{\prime}, s^{\prime}\right)$. Consequently, $F(x)+a_{0}|x|^{2} / 2 k$ is continuous in $\bar{B}\left(y^{\prime}, s^{\prime}\right)$, and we conclude that the set of points $Z \subset B\left(x_{0}, h_{0}\right)$ at which $F(x)+a_{0}|x|^{2} / 2 k$ is not continuous must be nondense (nowhere dense).

We next observe that $Z$ contains no isolated points. For if $z_{0}$ were an isolated point of $Z$, then there would exist $s_{0}>0$ such that $F(x)$ $+a_{0}|x|^{2} / 2 k$ would be harmonic in the punctured $k$-ball $B\left(z_{0}, 2 s_{0}\right)-z_{0}$. Therefore by (37), (40), (42), and the mean-value theorem for harmonic functions, for $x$ in $B\left(z_{0}, h\right)-z_{0}$ and $0<h<s_{0}$

$$
\begin{aligned}
\mid F\left(z_{0}\right) & +a_{0}\left|z_{0}\right|^{2} / 2 k-F(x)-a_{0}|x|^{2} / 2 k \mid \\
= & \left.\left|F\left(z_{0}\right)+a_{0}\right| z_{0}\right|^{2} / 2 k \\
& -\left\{F_{\left|z_{0}-x\right|}(x)+a_{0} \int_{B\left(x,\left|z_{0}-x\right|\right)}|y|^{2} d y /\left|B\left(x,\left|z_{0}-x\right|\right)\right| 2 k\right\} \mid \\
\leqq & \left|F\left(z_{0}\right)-F_{|z,-x|}(x)\right| \\
& +\left|a_{0}\right|\left|\int_{B\left(x,\left|z_{0}-x\right|\right)}\left(|y|^{2}-\left|z_{0}\right|^{2}\right) d y\right| /\left|B\left(x,\left|z_{0}-x\right|\right)\right| 2 k \\
\leqq & \left|F\left(z_{0}\right)-F_{\left|z_{0}-x\right|}(x)\right|+o(1) \\
\leqq & \left|F\left(z_{0}\right)-F\left(z_{0},\left|z_{0}-x\right|\right)\right|+\left|F\left(z_{0},\left|z_{0}-x\right|\right)-F_{\left|z_{0}-x\right|}\left(z_{0}\right)\right| \\
& +\left|F_{\left|z_{0}-x\right|}\left(z_{0}\right)-F_{\left|z_{0}-x\right|}(x)\right|+o(1) \\
\leqq & o(1)+\alpha_{1}(h)+\alpha_{2}(h) \\
\leqq & o(1) .
\end{aligned}
$$

Consequently, $\lim _{x \rightarrow \varepsilon_{0}} F(x)+a_{0}|x|^{2} / 2 k=F\left(z_{0}\right)+a_{0}\left|z_{0}\right|^{2} / 2 k$. We conclude that $Z$ can contain no isolated points.

Next, let $\bar{Z}$ designate the closure of $Z$. Since $|f(x, t)| \rightarrow 0$ for $x$ in 
$\bar{Z}$ and since $\bar{Z}$ is a perfect, we can obtain once again from the Baire category theorem and (44) that if $\bar{Z}$ is a nonempty set that there exists a $z_{0}$ in $Z$ and an $s_{0}>0$ with $\bar{B}\left(z_{0}, 2 s_{0}\right) \subset B\left(x_{0}, h_{0}\right)$ and a constant $K$ such that

$$
|f(z, t)| \leqq K+1 \quad \text { for } z \text { in } \bar{Z} \bar{B}\left(z_{0}, 2 s_{0}\right) .
$$

We propose to show that $F(x)+a_{0}|x|^{2} / 2 k$ is actually continuous at $z_{0}$, and consequently that $Z$ is the empty set, i.e. $F(x)+a_{0}|x|^{2} / 2 k$ is continuous in $B\left(x_{0}, h_{0}\right)$.

Employing the same technique used in establishing (37), we see from (45), that $f_{1}(z, t)$ and $F(z, t)$ converge uniformly as $t \rightarrow 0$ for $z$ in $\bar{Z} \bar{B}\left(z_{0}, 2 s_{0}\right)$, and consequently since this latter set is closed that $F(x)$ is continuous for $x$ restricted to $\bar{Z} \bar{B}\left(z_{0}, 2 s_{0}\right)$. Therefore given an $\epsilon>0$, choose $s_{1}$ such that $0<s_{1}<s_{0}$ and such that

$$
\left|F(z)-F\left(z_{0}\right)\right|<\epsilon \quad \text { for } z \text { in } \overline{Z B}\left(z_{0}, s_{1}\right) .
$$

Next, using (40), (42), and (45) choose $s_{2}$ such that

$$
\begin{aligned}
\alpha_{1}(s)<\epsilon \text { and } \alpha_{2}(s) & <\epsilon \quad \text { for } 0<s<s_{2} ; \\
\left|a_{0}\right|\left(2\left|z_{0}\right|+3 s_{2}\right) s_{2} & <\epsilon ; \\
|F(z, s)-F(z)| & <\epsilon \quad \text { for } 0<s<s_{2} \text { and } z \operatorname{in} \bar{Z} B\left(z_{0}, 2 s_{0}\right) ; \\
2 s_{2} & <s_{1} .
\end{aligned}
$$

We propose to show that

$$
\left|F(x)-F\left(z_{0}\right)\right|<5 \epsilon \quad \text { for } x \text { in } B\left(z_{0}, s_{2}\right) .
$$

If $x$ is in $B\left(z_{0}, s_{2}\right)$ and $x$ is in $\bar{Z}$, then (48) holds by (46) and (47). We can therefore suppose that $x$ is in $B\left(z_{0}, s_{2}\right)$ and $x$ is not in $\bar{Z}$. Let $z^{\prime}$ be the closest point in $\bar{Z}$ (or one of the closest if more than one exists) to $x$. Then $\left|z^{\prime}-x\right|=s_{3}<s_{2}$ and $F(y)+a_{0}|y|^{2} / 2 k$ is a harmonic function in $B\left(x, s_{3}\right)$. Therefore,

$$
\begin{aligned}
& \left|F(x)-F\left(z_{0}\right)\right| \leqq\left|F(x)-F\left(z^{\prime}\right)\right|+\left|F\left(z^{\prime}\right)-F\left(z_{0}\right)\right| \\
& \leqq\left|F_{s_{3}}(x)-F\left(z^{\prime}\right)\right|+\left|a_{0}\right| \\
& \cdot(2 k)^{-1}\left|\int_{B\left(x, s_{3}\right)}\left[|y|^{2}-|x|^{2}\right] d y\right|\left|B\left(x, s_{3}\right)\right|^{-1} \\
& +\left|F\left(z^{\prime}\right)-F\left(z_{0}\right)\right| \\
& \leqq\left|F_{8_{3}}(x)-F\left(z^{\prime}\right)\right|+2 \epsilon \text {, }
\end{aligned}
$$

by (46) and (47).

But once again by (47), 


$$
\begin{aligned}
\left|F_{s_{3}}(x)-F\left(z^{\prime}\right)\right| \leqq & \left|F_{s_{3}}(x)-F_{s_{3}}\left(z^{\prime}\right)\right|+\left|F_{s_{3}}\left(z^{\prime}\right)-F\left(z^{\prime}, s_{3}\right)\right| \\
& +\left|F\left(z^{\prime}, s_{3}\right)-F\left(z^{\prime}\right)\right| \\
\leqq & \alpha_{2}\left(s_{3}\right)+\alpha_{1}\left(s_{3}\right)+\left|F\left(z^{\prime}, s_{3}\right)-F\left(z^{\prime}\right)\right| \leqq 3 \epsilon .
\end{aligned}
$$

This last part coupled with (49) gives us (48). We conclude first that $F$ is continuous at $z_{0}$, next that $Z$ is empty, and finally that $F(x)+a_{0}|x|^{2} / 2 k$ is harmonic in $B\left(x_{0}, h_{0}\right)$.

Since $B\left(x_{0}, h_{0}\right)$ was any open $k$-ball in $E_{k}$ whose closure does not contain a point of the form $2 \pi m$, we have that $F(x)+a_{0}|x|^{2 / 2 k}$ is harmonic in $E_{k}-U_{m}\{2 \pi m\}$.

To complete the proof of the theorem, we need the following lemma.

Lemma 8. For $t>0$, set

$$
H_{0}(x, t)=\sum_{m \neq 0} e^{i(m, x)-|m| t /|m|^{2},}
$$

and for $j=1, \cdots, k$,

$$
H_{j}(x, t)=\sum_{m \neq 0} i m_{j} e^{i(m, x)-|m| t} /|m|^{2} .
$$

Then for $j=0,1, \cdots, k$,

$\lim _{t \rightarrow 0} H_{j}(x, t)=H_{j}(x)$ exists and is finite for $x \neq 2 \pi m, H_{j}(x)$ is in $L^{1}$ on $T_{k}$,

and

$$
\lim _{t \rightarrow 0} \int_{T_{k}}\left|H_{j}(x, t)-H_{j}(x)\right| d x=0
$$

Set

$$
\begin{aligned}
& \Phi(x)=(2 \pi)^{k}\left[\omega_{k-1}(k-2)\right]^{-1}|x|^{-(k-2)} \quad \text { for } x \text { in } T_{k}-0 \text { and } \quad k \geqq 3 \\
& =(2 \pi) \log |x|^{-1} \quad \text { for } x \text { in } T_{2}-0 \text { and } k=2 \text {, }
\end{aligned}
$$

and extend $\Phi(x)$ by periodicity to all of $E_{k}$. Then

$$
\lim _{|x| \rightarrow 0}\left[H_{0}(x)-\Phi(x)\right] \text { exists and is finite }
$$

and

$$
B_{0}(x)-|x|^{2} / 2 k \text { is harmonic in } E_{k}-\underset{m}{\bigcup}\{2 \pi m\} .
$$

Furthermore, for $j=1, \cdots, k$,

$$
\lim _{|x| \rightarrow 0} H_{j}(x)+(2 \pi)^{k} x_{j} / \omega_{k-1}|x|^{k} \text { exists and is finite }
$$

and 


$$
H_{j}(x) \text { is harmonic in } E_{k}-\bigcup_{m}\{2 \pi m\} \text {. }
$$

Let us temporarily assume the validity of the lemma, complete the proof of the theorem, and then prove the lemma.

We have that $F(x)+a_{0}|x|^{2} / 2 k$ is harmonic in $E_{k}-U_{m}\{2 \pi m\}$ and that $F(x)$ is a periodic function in $L^{2}$ on $E_{k}$. It is well known [16, p. 269] that under these conditions there is a function $U(x)$ which is harmonic in $B(0,1)$ such that (with $\Phi(x)$ as in Lemma 8)

$$
F(x)+a_{0}|x|^{2} / 2 k=U(x)+c_{0}^{\prime} \Phi(x)+\sum_{n=k-1}^{\infty} Y_{n+2-k}\left(x|x|^{-1}\right)|x|^{-n}
$$

for $x$ in $B(0,1)-0$ where $Y_{n}$ is a surface spherical harmonic of order $n$ and the series on the right side of (50) is uniformly convergent outside of any sphere containing the origin. (Note that in two dimensions, using polar coordinates $(r, \theta), Y_{n}$ is a linear combination of $\sin n \theta$ and $\cos n \theta$.) The fact that $F(x)$ and $\Phi(x)$ are in $L^{1}$ on $B(0,1)$ immediately implies that $Y_{n}(x)$ is identically zero for $n \geqq k$. We conclude, consequently, from (50) that

$$
\begin{aligned}
F(x)+a_{0}|x|^{2} / 2 k & \\
& =U(x)+c_{0}^{\prime} \Phi(x)+\sum_{j=1}^{k} c_{j}^{\prime} x_{j}|x|^{-k} \quad \text { for } x \text { in } B(0,1)-0,
\end{aligned}
$$

and, therefore, from Lemma 8 that there are constants $c_{0}, c_{1}, \cdots, c_{k}$ such that

$$
\lim _{|x| \rightarrow 0} F(x)+\left(a_{0}-c_{0}\right)|x|^{2} / 2 k+\sum_{j=0}^{k} c_{j} H_{j}(x)
$$

$=\beta$ where $\beta$ is finite-valued.

Setting

$$
\begin{aligned}
V(x)= & F(x)+\left(a_{0}-c_{0}\right)|x|^{2} / 2 k \\
& +\sum_{j=0}^{k} c_{j} H_{j}(x) \quad \text { for } x \text { in } E_{k}-\bigcup_{m}\{2 \pi m\} . \\
V(2 \pi m)= & \beta+\left(a_{0}-c_{0}\right)|2 \pi m|^{2} / 2 k,
\end{aligned}
$$

we obtain from the periodicity of $F(x)$ and $H_{j}(x)$ and (52) that $V(x)$ is continuous in $E_{k}$ and consequently from Lemma 8 and the properties already established for $F$, that $V(x)$ is harmonic in $E_{k}$. But $V(x)-\left(a_{0}-c_{0}\right)|x|^{2} / 2 k$ is periodic and therefore bounded in $E_{k}$. We infer, then, from well-known properties of harmonic functions [16, pp. 252-253] that $V(x)$ must be a polynomial of degree less than or 
equal to two. However, the only way that the polynomial $V(x)$ $-\left(a_{0}-c_{0}\right)|x|^{2} / 2 k$ can be periodic is if it is a constant. We conclude from (52) that there is a constant $K$ such that

$$
F(x)+\sum_{j=0}^{k} c_{j} H_{j}(x)=K \quad \text { for } x \text { in } E_{k}-\bigcup_{m}\{2 \pi m\} .
$$

From the definition of $F(x)$ and Lemma 8, we obtain from (53) that

$$
a_{m}+c_{0}=-\sum_{j=1}^{k} i c_{j} m_{j} \quad \text { for } m \neq 0 .
$$

By (i) of the theorem, $a_{m}+c_{0}=o(|m|)$ as $m \rightarrow \infty$. We conclude from (50) that $c_{j}=0$ for $j=1, \cdots, k$ and therefore from (50) that $a_{m}=-c_{0}$. But by (i) of the theorem $\sum_{R_{\leq}|m| \leq R+1}\left|a_{m}\right|=o(R)$ as $R \rightarrow \infty$. On the other hand the number of lattice points in the annulus $R \leqq|m| \leqq R+1$ is $O\left(R^{k-1}\right)$ as $R \rightarrow \infty$. $c_{0}$ must therefore be zero, and consequently $a_{m}=0$ for $m \neq 0$. But then by (ii) of the theorem, $a_{0}=0$, and the theorem is established.

We now prove Lemma 8 (see $[24$, p. 500$]$ ).

We first show that there exists a continuous periodic function $\psi(x)$ such that

$$
\begin{aligned}
\hat{\Phi}(m)-\hat{\psi}(m) & =|m|^{-2} \quad \text { for } m \neq 0, \\
\hat{\Phi}(0)-\hat{\psi}(0) & =0 . \\
\sum_{m}|\hat{\psi}(m)| & <\infty .
\end{aligned}
$$

Observing from Green's second identity that for $k \geqq 3$ and $m \neq 0$

$$
\begin{aligned}
\int_{T_{k}-B(0, \epsilon)}|x|^{-(k-2)} e^{-i(m, x)} d x & \\
& =\int_{\partial\left[T_{k}-B(0, e)\right]} e^{-i(m, x)}|m|^{-2 \partial}|x|^{-(k-2) / \partial n d S+o(1)}
\end{aligned}
$$

with a similar remark holding for log $|x|^{-1}$, we obtain in a manner similar to the establishment of (5) that for $m \neq 0$

$$
\begin{gathered}
\hat{\Phi}(m)=|m|^{-2} \\
+d_{k}|m|^{-2} \sum_{j=1}^{k} \int_{T_{k-1}} \frac{\exp \left[i\left(m, x_{1}+\cdots+m_{j} x_{j}^{*}+\cdots+m_{k} x_{k}\right)\right]}{\left[x_{1}^{2}+\cdots+\pi^{2}+\cdots+x_{k}^{2}\right]^{k / 2}}
\end{gathered}
$$


where $d_{k}$ is a constant depending on $k$ but not on $m$ and $m_{j} x_{j}^{*}$ stands for the deletion of $m_{j} x_{j}$.

We now define $\hat{\psi}(m)$ to be the second expression on the right in (57) if $m \neq 0$ and $\hat{\psi}(0)=\hat{\Phi}(0) . \hat{\psi}(m)$ consequently satisfies (55). To show (56), we observe that for $m_{1} \neq 0$

$$
\begin{aligned}
\int_{-\pi}^{\pi} e^{i m_{1} x_{1}}\left[x_{1}^{2}+\cdots+\right. & \left.\pi^{2}+\cdots+x_{k}^{2}\right]^{-k / 2} d x_{1} \\
& =k \int_{-\pi}^{\pi} \frac{\sin m_{1} x_{1} x_{1}}{m_{1}\left[x_{1}^{2}+\cdots+\pi^{2}+\cdots x_{k}^{2}\right]^{(k+2) / 2}} d x_{1}
\end{aligned}
$$

and obtain consequently that there is a constant $d_{k}^{\prime}$ such that

$$
\begin{gathered}
|\hat{\psi}(m)| \leqq d_{k}^{\prime}(|m|+1)^{-2} \sum_{j=1}^{k}\left(\left|m_{1}\right|+1\right)^{-1} \ldots \\
\cdot\left(\left|m_{j}\right|+1\right)^{-1 *} \ldots\left(\left|m_{k}\right|+1\right)^{-1} .
\end{gathered}
$$

On the other hand,

$$
\begin{aligned}
& {\left[\left(\left|m_{1}\right|+1\right) \cdots\left(\left|m_{k}\right|+1\right)\right]^{(k+1) / k}} \\
& \leqq(|m|+1)^{2}\left(\left|m_{1}\right|+1\right) \cdots\left(\left|m_{j}\right|+1\right)^{*} \cdots\left(\left|m_{k}\right|+1\right) .
\end{aligned}
$$

(58) and (59) imply that

$$
\sum_{m}|\hat{\psi}(m)| \leqq k d_{k}^{\prime} \sum_{m}\left[\left(\left|m_{1}\right|+1\right) \cdots\left(\left|m_{k}\right|+1\right)\right]^{-(k+1) / k}<\infty \text {, }
$$

and (56) is established.

We now define $\psi(x)=\sum_{m} \hat{\psi}(m) e^{i(m, x)}$ and obtain that $\psi(x)$ is a continuous periodic function and furthermore that

$$
\Phi(x)-\psi(x) \sim \sum_{m \neq 0} e^{i(m, x)} /|m|^{2}
$$

Setting $H_{0}(x, t)=\sum_{m \neq 0} e^{i(m, x)-|m| t} /|m|^{2}$, it now follows immediately from Theorem 2 that $\lim _{t \rightarrow 0} H_{0}(x, t)=H_{0}(x)$ exists and is finite for $x$ in $E_{k}-U_{m}\{2 \pi m\}$ and furthermore that for $x$ in $E_{k}-U_{m}\{2 \pi m\}$, $H_{0}(x)=\Phi(x)-\psi(x)$. But $\psi(x)$ is a continuous function. Consequently, $\lim _{|x| \rightarrow 0} H_{0}(x)-\Phi(x)$ exists and is finite.

To complete the part of Lemma 8 dealing with $H_{0}(x)$, we have to show that $\lim _{t \rightarrow 0} H_{0}(x, t)=H_{0}(x)$ in the $L^{1}$-norm on $T_{k}$ and that $H_{0}(x)-|x|^{2} / 2 k$ is harmonic in $E_{k}-U_{m}\{2 \pi m\}$.

In order to accomplish this, we look at the function $P(x, t)$ $=\sum_{m} e^{i(m, x)-|m| t}$ for $t>0$. Using the calculations in Theorem 2 , we see that 


$$
(2 \pi)^{-k} \int_{E_{k}} e^{-i(x, y)-|y| t} d y=b_{k}\left[t^{2}+|x|^{2}\right]^{-(k+1) / 2}
$$

and consequently that

$$
\int_{E_{k}} b_{k} t\left[t^{2}+|x|^{2}\right]^{-(k+1) / 2} e^{i(y, x)} d x=e^{-|y| t} .
$$

But then by Remark 1, (30),

$$
b_{k}(2 \pi)^{k} t \sum_{m}\left[t^{2}+|x-2 \pi m|^{2}\right]^{-(k+1) / 2}=P(x, t) .
$$

We conclude that

(62) $\lim _{t \rightarrow 0} P(x, t)=0$ uniformly for $x$ in $T_{k}-B(0, \epsilon) \quad$ for $\epsilon>0$.

We next establish the following remark:

REMARK 2. Let $f$ be a function in $L^{1}$ on $T_{k}$ and extended by periodicity to all of $E_{k}$. For $t>0$, set $A_{t}(f, x)=\sum_{m} \hat{f}(m) e^{i(m, x)}-|m| t$. Then

$$
\lim _{t \rightarrow 0} \int_{T_{k}}\left|A_{t}(f, x)-f(x)\right| d x=0 .
$$

Observing that $A_{t}(f, x)=(2 \pi)^{-k} \int_{T_{k}} f(x-y) P(y, t) d y$ and that $(2 \pi)^{-k} \int_{T_{h}} P(y, t) d y=1$, we see that

$$
A_{t}(f, x)-f(x)=(2 \pi)^{-k} \int_{T_{k}}[f(x-y)-f(x)] P(y, t) d y
$$

and consequently that

$$
\begin{aligned}
& \int_{T_{k}}\left|A_{t}(f, x)-f(x)\right| d x \\
& \quad \leqq(2 \pi)^{-k} \int_{T_{k}} P(y, t)\left[\int_{T_{k}}|f(x-y)-f(x)| d x\right] d y \\
& \quad \leqq(2 \pi)^{-k} \int_{B(0, \delta)} P(y, t)\left[\int_{T_{k}}|f(x-y)-f(x)| d x\right] d y+o(1)
\end{aligned}
$$

by (62) for $0<\delta<1$.

Using the fact that $\lim _{|y| \rightarrow 0} \int_{T_{b}}|f(x-y)-f(x)| d x=0$, Remark 2 follows immediately from (63).

Since $H_{0}(x)=\Phi(x)-\psi(x)$ is in $L^{1}$ on $T_{k}$, it follows from Remark 2 that $\int_{T_{k}}\left|H_{0}(x, t)-H_{0}(x)\right| d x \rightarrow 0$ as $t \rightarrow 0$. 
Next, let $x_{0}$ be a point in $E_{k}-\bigcup_{m}\{2 \pi m\}$. Then there exists $\epsilon>0$ such that $H_{0}(x)$ is continuous in $B\left(x_{0}, \epsilon\right)$ since it is equal to $\Phi(x)$ $-\psi(x)$ in this $k$-ball. By Lemma 7 and (62) $\Delta_{*}\left[H_{0}(x)-|x|^{2} / 2 k\right] \leqq 0$ and $0 \leqq \Delta^{*}\left[H_{0}(x)-|x|^{2} / 2 k\right]$ for $x$ in $B\left(x_{0}, \epsilon\right)$.

But then by $\left[21\right.$, p. 14], $H_{0}(x)-|x|^{2} / 2 k$ is harmonic in $B\left(x_{0}, \epsilon\right)$, and all the statements concerning $H_{0}(x)$ in Lemma 8 are established.

To establish the rest of the statements in Lemma 8 , we note that for $\epsilon>0$ and small that $\psi(x)+|x|^{2} / 2 k$ is harmonic in $B(0, \epsilon)-0$, and furthermore that $\psi(x)+|x|^{2} / 2 k$ is continuous in $B(0, \epsilon)$. Consequently, $\psi(x)+|x|^{2} / 2 k$ is harmonic in $B(0, \epsilon)$, and therefore $\lim _{|x| \rightarrow 0} \partial \psi(x) / \partial x_{j}$ exists and is finite for $j=1, \cdots, k$. But then

$$
\lim _{|x| \rightarrow 0} \partial H_{0}(x) / \partial x_{j}+(2 \pi)^{k} x_{j} / \omega_{k-1}|x|^{k} \text { exists and is finite. }
$$

Also, from what has already been established, we know that $\partial H_{0}(x) / \partial x_{j}$ is harmonic in $E_{k}-U_{m}\{2 \pi m\}$. Furthermore from (64), we observe that $\partial H_{0}(x) / \partial x_{j}$ is in $L^{1}$ on $T_{k}$. Consequently, it follows from Theorem 2 that we shall have established all of Lemma 8 once we show that

$$
\begin{aligned}
\int_{T_{k}} \partial H_{0}(x) / \partial x_{j} d x & =0, \\
\int_{T_{k}} e^{-i(m, x)} \partial H_{0}(x) / \partial x_{j} & =(2 \pi)^{k} i m_{j}|m|^{-2} \quad \text { for } m \neq 0 .
\end{aligned}
$$

To establish (65), let $\mathrm{v}(x)$ be the vector field defined in $T_{k}-0$ whose $j$ th component is $H_{0}(x)$ and whose other $(k-1)$ components are zero. Then by the divergence theorem the left side of (65) is equal to

$$
\lim _{\epsilon \rightarrow 0} \int_{\partial\left[T_{k}-B(0, \epsilon)\right]}(\mathrm{v}, \mathrm{n}) d S .
$$

But $H_{0}(x)$ is a periodic function. Consequently $\int_{\partial T_{k}}(\mathrm{v}, \mathrm{n}) d S=0$. Therefore the left side of (65) is majorized by

$$
\limsup _{e \rightarrow 0} \max _{|x|=\bullet}\left|H_{0}(x)\right| \epsilon^{k-1} \omega_{k-1} .
$$

However we already have shown that $H_{0}(x)=o\left(|x|^{1-k}\right)$ as $|x| \rightarrow 0$. Consequently, (65) is established.

To show that (66) holds, we now let $\mathrm{v}(x)$ be the vector field defined in $T_{k}-0$ whose $j$ th component is $e^{-i(m, x)} H_{0}(x)$ and whose other $(k-1)$ components are zero. Then the same reasoning as above shows that 


$$
\lim _{\epsilon \rightarrow 0} \int_{\partial\left[T_{k}-B(0, \epsilon)\right]}(\mathrm{v}, \mathrm{n}) d S=0 .
$$

On the other hand, the divergence theorem gives

$$
\int_{\partial\left[T_{k}-B(0, e)\right]}(\mathrm{v}, \mathrm{n}) d S=\int_{T_{k}-B(0, e)} \partial\left[e^{-i(m, x)} H_{0}(x)\right] / \partial x_{j} d x .
$$

We conclude that

$$
\begin{aligned}
\int_{T_{k}} e^{-i(m, x)} \partial H_{0} / \partial x_{j} d x & =\int_{T_{k}} i m_{j} H_{0}(x) e^{-i(m, x)} d x \\
& =i m_{j}(2 \pi)^{k}|m|^{-2} \quad \text { for } m \neq 0 .
\end{aligned}
$$

(66) is established, and the proof of the lemma is complete.

5. Conjugate Fourier series. In this section, we shall describe some results in the theory of conjugate multiple Fourier series and the related topic of analyticity in several variables. The conjugate series will be defined by means of the Calderon-Zygmund kernel. (Other names associated with the development of the singular kernel involved are Beurling, Mihlin, and M. Riesz. See [8, p. 137].)

In order to place the theory in its proper perspective let us briefly review some aspects of the theory of 1-dimensional conjugate Fourier series. If $g(x)$ is a function in $L^{1}$ on $E_{1}$, then $\tilde{g}(x)=$ P.V. $g_{*} x^{-1}$ is defined to be the Hilbert transform of $g$ where

$$
\text { P.V. } g_{*} x^{-1}=\lim _{h \rightarrow 0} \pi^{-1} \int_{h}^{\infty}[g(x-y)-g(x+y)] / y d y .
$$

Now this limit exists almost everywhere, and it is well known that if $\tilde{g}(x)$ is in $L^{1} \cap L^{2}$ then $\tilde{g}^{\Upsilon}(x)=-i \operatorname{sgn} x \hat{g}(x)$ (where $\operatorname{sgn} x=1$ if $x>0,-1$ if $x<0$, and 0 if $x=0$ ). However, even if $\tilde{g}(x)$ is not in $L^{1} \cap L^{2}$, we still obtain $[34$, p. 147] that for almost every $x$

$$
\lim _{R \rightarrow \infty} \int_{-R}^{R}-i \operatorname{sgn} y \hat{g}(y) e^{i x y}(1-|y| / R)^{\alpha}=\tilde{g}(x) \quad \text { for } \alpha>0 .
$$

To pass from Fourier integrals to Fourier series, we first observe that $[37$, p. 73$]$

$$
\frac{1}{2} \cot \frac{x}{2}=x^{-1}+\sum_{m=-\infty}^{\infty}\left[(x+2 \pi m)^{-1}-(2 \pi m)^{-1}\right] \quad \text { for } x \neq 2 \pi m \text {. }
$$

Next, we observe that if $f(x)$ is in $L^{1}$ on $T_{1}$ and extended by periodic- 
ity to all of $E_{1}$, then $\tilde{f}(x)=$ P.V. $f_{*} 2^{-1} \cot x / 2$ is defined to be the conjugate function of $f$ where

$$
\begin{aligned}
& \text { P.V. } f_{*} 2^{-1} \cot x / 2 \\
& \qquad \lim _{h \rightarrow 0}(\pi)^{-1} \int_{h}^{\pi}[f(x-y)-f(x+y)] 2^{-1} \cot y / 2 d y .
\end{aligned}
$$

Now, it is well known [37, p. 131] that this limit exists almost everywhere, and that if $\tilde{f}(x)$ is in $L^{1}$, then $\tilde{f}^{\wedge}(m)=-i \operatorname{sgn} m \hat{f}(m)$. However, even if $\tilde{f}(x)$ is not in $L^{1}$, we still obtain that for almost every $x$, $\sum_{-\infty}^{\infty}-i \operatorname{sgn} m \hat{f}(m) e^{i m x}$ is Cesaro summable of order $\alpha, \alpha>0$, to $\tilde{f}(x)$ for almost every $x$.

We note also that if the limit on the right side of (67) exists then

$$
\begin{aligned}
\lim _{h \rightarrow 0} \int_{h}^{\pi}[f(x-y)-f(x+y)] 2^{-1} \cot y / 2 d y \\
=\lim _{h \rightarrow 0} \lim _{\lambda \rightarrow \infty} \int_{h}^{\lambda}[f(x-y)-f(x+y)] y^{-1} d y .
\end{aligned}
$$

Now the function $x^{-1}=\operatorname{sgn} x|x|^{-1}$, and $\operatorname{sgn} 1+\operatorname{sgn}-1=0$. We generalize this function to $k$-space by means of the kernel

$$
K(x)=W(x /|x|)|x|^{-k} \quad \text { for } x \neq 0
$$

where $W(x /|x|)$ satisfies the additional conditions:

$$
\begin{aligned}
& \text { there is a constant } \mu \text { such }\left|W\left(\xi_{1}\right)-W\left(\xi_{2}\right)\right| \leqq \mu\left|\xi_{1}-\xi_{2}\right|^{\beta} \\
& \beta>0, \text { for } \xi_{1} \text { and } \xi_{2} \text { on } C(0,1) \text { the unit }(k-1) \text { sphere in } E_{k}
\end{aligned}
$$

and

$$
\int_{C(0,1)} W(\xi) d c(\xi)=0
$$

where $d c(\xi)$ is the natural volume element on $C(0,1)$.

A kernel $K(x)$ of this type is called a Calderón-Zygmund kernel and is clearly a generalization of the Hilbert kernel $x^{-1}$ in 1-dimension. The generalization persists in the sense that if $g(x)$ is a function in $L^{1}$ on $E_{k}$, then $\tilde{g}(x)=(2 \pi)^{-k}$ P.V. $g_{*} K$ is defined to be the CalderónZygmund transform of $g$ where

$$
\text { P.V. } g_{*} K(x)=\lim _{\epsilon \rightarrow 0} \int E_{k-B(0, \epsilon)} g(x-y) K(y) d y .
$$

It is shown in $[8$, p. 118] that this limit exists almost everywhere. 
It is also shown in [8] that the principal-valued Fourier transform $\hat{K}(y)$ of the Calderón-Zygmund kernel $K(x)$ exists for every $y$ where

$$
\hat{K}(y)=\lim _{\epsilon \rightarrow 0} \lim _{\lambda \rightarrow \infty}(2 \pi)^{-k} \int_{B(0, \lambda)-B(0, e)} e^{-i(x, y)} K(y) d y .
$$

This limit is specifically evaluated in $[27$, p. 69] where it is shown that if $W(\xi)$ has the surface spherical harmonic expansion

$$
W(\xi) \sim \sum_{n=1}^{\infty} Y_{n}(\xi)
$$

then for $y \neq 0$,

$$
\hat{K}(y)=\sum_{n=1}^{\infty}(-i)^{n} Y_{n}(y /|y|) \Gamma(n / 2) / 2^{k} \Gamma[(n+k) / 2] \pi^{k / 2},
$$

where the series in (66) converges absolutely uniformly.

(The special case of $(71)$ when $W(\xi)=Y_{n}(\xi)$ was first evaluated in [5]. See also $[9$, p. 261].)

Next, we define $K^{*}(x)$ the periodic analogue of $K(x)$ as follows:

$$
K^{*}(x)=K(x)+\sum_{m \neq 0}[K(x+2 \pi m)-K(2 \pi m)] \quad \text { for } x \neq 2 \pi m .
$$

In [9], it is shown that the series in (72) converges absolutely uniformly for $x$ in $T_{k}$ (with a similar fact holding for $x$ in any bounded domain after a finite number of terms of the series are deleted) and that for $x \neq 2 \pi m, K^{*}(x+2 \pi m)=K^{*}(x)$.

If $f(x)$ is in $L^{1}$ on $T_{k}$ and extended by periodicity to all of $E_{k}$, it then follows that

$$
\tilde{f}(x)=\lim _{\epsilon \rightarrow 0}(2 \pi)^{-k} \int_{T_{k}-B(0, \epsilon)} f(x-y) K^{*}(y) d y
$$

exists and is finite for almost every $x$.

It is easy to see that if $\int_{T_{k}} f(y) d y=0$ and if the limit on the right side of (73) exists and is finite then

$$
\tilde{f}(x)=\lim _{\epsilon \rightarrow 0} \lim _{\lambda \rightarrow \infty}(2 \pi)^{-k} \int_{B(0, \lambda)-B(0, \epsilon)} f(x-y) K(y) d y .
$$

Next, defining the principal-valued $m$ th Fourier coefficient $\hat{K}^{*}(m)$ as $\lim _{\epsilon \rightarrow 0}(2 \pi)^{-k} \int_{T_{k-B(0, \epsilon)}} e^{-i(m, x)} K^{*}(x) d x$ it is shown in $[9$, p. 257] that for $m \neq 0, \hat{K}^{*}(m)=\hat{K}(m)$. It is, furthermore, shown in [9, p. 259] that if $\tilde{f}(x)$ is in $L^{1}$ on $T_{k}$, then $\tilde{f}^{\wedge}(m)=\hat{f}(m) \hat{K}(m)$ for $m \neq 0$. Consequently, 
bearing the 1-dimensional situation in mind, it is natural to define $\tilde{S}[f]$, the conjugate series of $S[f]$, as

$$
\tilde{S}[f]=\sum_{m \neq 0} \hat{K}(m) \hat{f}(m) e^{i(m, x)} .
$$

We shall say that $K(x)=W(x /|x|) /|x|^{k}$ is a kernel in class $C^{n}$ if $W(\xi)$ is a function in class $C^{n}$ on the $(k-1)$ sphere $C(0,1)$. The following companion results to Theorems 1 and 2 of this survey were then obtained by Shapiro in [27, pp. 43-44].

THEOREM 8. Let $f$ be in $L^{1}$ on $T_{k}$ and extended by periodicity to all of $E_{k}$, and let $S[f]=\sum_{m} \hat{f}(m)^{i(m, x)}$. Furthermore let $K(x)$ be a CalderónZygmund kernel in class $C^{k+4}$ and let $\hat{K}(y)$ be its principal-valued Fourier transform. Set

$$
\tilde{\sigma}_{R}^{\alpha}(f, x)=\sum_{1 \leq|m| \leq R} \hat{K}(m) \hat{f}(m) e^{i(m, x)}\left(1-|m|^{2} / R^{2}\right)^{\alpha}
$$

with $(k-1) / 2<\alpha \leqq(k-1)+1 / 2$ if $k$ is even and $(k-1) / 2<\alpha$ $\leqq(k-1) / 2+1$ if $k$ is odd. Suppose that

$$
\left|B\left(x_{0}, h\right)\right|^{-1} \int_{B\left(x_{0}, h\right)}\left|f(x)-f\left(x_{0}\right)\right| d x \rightarrow 0 \quad \text { as } h \rightarrow 0 .
$$

Then

$$
\lim _{R \rightarrow \infty}\left[\tilde{\sigma}_{R}^{\alpha}\left(f, x_{0}\right)-\lim _{\lambda \rightarrow \infty} \int_{B(0, \lambda)-B\left(0, R^{-1}\right)} f\left(x_{0}-y\right) K(y) d y\right]=0 .
$$

For the 1-dimensional analogue of the above theorem, see [37, p. 95]. We obtain immediately from Theorem 8, (73), and (74) that if $\int_{T_{k}} f(y) d y=0$, then for almost every $x, \tilde{\sigma}_{R}^{\alpha}(f, x) \rightarrow \tilde{f}(x)$ for $\alpha>(k-1) / 2$.

The following companion result for Abel summability (Theorem 2) is also obtained:

Theorem 9. Let $f$ be in $L^{1}$ on $T_{k}$ and extended by periodicity to all of $E_{k}$ and let $S[f]=\sum_{m} \hat{f}(m) e^{i(m, x)}$. Furthermore, let $K(x)$ be a Calderón-Zygmund kernel in class $C^{k / 2}$ or $C^{(k+1) / 2}$ according as to whether $k$ is even or odd. Set $\tilde{A}_{t}(f, x)=\sum_{m \neq 0} \hat{f}(m) \hat{K}(m) e^{i(m, x)-|m| t}$ for $t>0$. Suppose that $\left|B\left(x_{0}, h\right)\right|^{-1} \int_{B\left(x_{0}, h\right)}\left|f(x)-f\left(x_{0}\right)\right| d x \rightarrow 0$ as $h \rightarrow 0$. Then

$$
\lim _{t \rightarrow 0}\left[\tilde{A}_{t}\left(f, x_{0}\right)-\lim _{\lambda \rightarrow \infty} \int_{B(0, \lambda)-B(0, t)} f\left(x_{0}-y\right) K(y) d y\right]=0 .
$$

Actually both Theorems 8 and 9 are proved for a wider class of Calderón-Zygmund kernels than indicated. In particular, Theorem 9 is true for kernels $K$ in class $C^{* \alpha}, \alpha>(k-1) / 2$. For the definition of 
the class $C^{* \alpha}$ and for the proofs of the above theorem, we refer the reader to [27, Chapter II].

Particular cases of what we have called the Calderón-Zygmund kernel were as mentioned earlier previously studied by other individuals. In particular, the kernel $K_{j}(x)=x_{j} /|x|^{k+1}(j=1, \cdots, k)$ which clearly satisfies (68), (69), and (70) is referred to as the Riesz kernel. (See [32, p. 30].)

The Riesz kernel is quite useful in studying generalizations of the notion of analyticity. In particular if $\mathbf{u}(x)=\left[u_{1}(x), \cdots, u_{k}(x)\right]$ is a $k$-dimensional vector field in class $C^{1}$ in a domain $D$ of $E_{k}$, we call $\mathrm{u}(x)$ an harmonic vector field in $D$ if

$$
\operatorname{div} \mathbf{u}=0 \text { and } \operatorname{curl} \mathrm{u}=0 \text { in } D
$$

or otherwise stated

$$
\sum_{j=1}^{k} \partial u_{j} / \partial x_{j}=0 \quad \text { and } \quad \partial u_{j_{1}} / \partial x_{j_{2}}=\partial u_{j_{2}} / \partial x_{j_{1}} \quad j_{1} \neq j_{2}
$$

It is clear that if $k=2$ and $u$ is an harmonic vector field in $D$, then $u_{2}+i u_{1}$ represents an analytic function in $D$.

With $K_{j}(x)$ as above, it follows from (71) that for $y \neq 0$

$$
\hat{K}_{j}(y)=-i y_{j} /|y| 2^{k} \Gamma[(k+1) / 2] \pi^{(k-1) / 2} .
$$

Consequently, if $S=\sum_{m} a_{m} e^{i(m, x)}$, it is natural to call

$$
\tilde{S}_{j}=-\sum_{m \neq 0} i m_{j}|m|^{-1} a_{m} e^{i(m, x)}, \quad j=1, \cdots, k,
$$

the $j$ th conjugate trigonometric series of $S$.

Letting $S(x, t)=\sum_{m} a_{m} e^{i(m, x)-|m| t}$ and

$$
\tilde{S}_{j}(x, t)=-\sum_{m \neq 0} i m_{j}|m|^{-1} a_{m} e^{i(m, x)-|m| t},
$$

we see that the vector field

$$
\mathrm{u}(x, t)=\left[\tilde{S}_{1}(x, t), \cdots, \tilde{S}_{k}(x, t), S(x, t)\right]
$$

is an harmonic vector field in the variables $\left(x_{1}, \cdots, x_{k}, t\right)$ for $t>0$.

We shall say that $S$ given above is a Fourier-Stieltjes series if there exist a countably additive set function $\mu$ defined on the bounded Borel sets of $E_{k}$ which is of bounded variation on $T_{k}$, which is periodic in the sense that $\mu(A+2 \pi m)=\mu(A)$ for every lattice point $m$ and bounded Borel set $A$, and which furthermore satisfies

$$
a_{m}=(2 \pi)^{-k} \int_{T_{k}} e^{-i(m, x)} d \mu(x) \quad \text { for every } m .
$$


Stein and Weiss [32, p. 55] have succeeded in generalizing the classical theorem of F. and M. Riesz in the context of the above terminology. It is a valid generalization in the sense that if one sets $k=1$ and replaces $e^{-t}$ by $r$ where $0<t$ and $0 \leqq r<1$, one gets back precisely the theorem of F. and M. Riesz. (See [37, p. 285].) The theorem of Stein and Weiss (though stated in terms of Fourier integrals in [32]) is the following:

TheOREM 10. Let $S=\sum_{m} a_{m} e^{i(m, x)}$ be a Fourier-Stieltjes series due to the set function $\mu$ and let $\widetilde{S}_{j}=-\sum_{m \neq 0} i m_{j}|m|^{-1} a_{m} e^{i(m, x)}$ be a FourierStieltjes series due to the set function $\mu_{j}(j=1, \cdots, k)$. Then $\mu$ and $\mu_{j}(j=1, \cdots, k)$ are absolutely continuous set functions. Furthermore if $f$ and $f_{j}$ are the functions in $L^{1}$ on $T_{k}$ corresponding respectively to $\mu$ and $\mu_{j}$, then there is a constant $\gamma$ depending on $k$ such that for almost every $x$

$$
f_{j}(x)=\lim _{\epsilon \rightarrow 0} \lim _{\lambda \rightarrow \infty} \gamma \int_{B(0, \lambda)-B(0, \epsilon)} f(x-y) y_{j}|y|^{-(k+1)} d y .
$$

There are other notions for generalizing the concept of analyticity than that mentioned above. In particular, there is a method associated with the concept of Dirichlet algebras (see for example [15, p. 54]). This concept leads to the following theorem of Bochner's [4, p. 718] (later reproved by Helson and Lowdenslager [14, p. 184] using different techniques) which is also a generalization of the F. and M. Riesz theorem:

THEOREM 11. Let $S=\sum_{m} a_{m} e^{i(m, x)}$ be a Fourier-Stieltjes series due to the complex-valued set function $\mu$. Suppose there exists a $\gamma$ with $1>\gamma$ $>0$ and an $x_{0} \neq 0$ such that $a_{m}=0$ if $\left(m, x_{0}\right)<\gamma|m|\left|x_{0}\right|$. Then $\mu$ is absolutely continuous.

6. Riemannian theory. In this section, we shall indicate some of the results in what is called the Riemannian theory of trigonometric series, i.e. the local aspects of trigonometric series.

We shall first look at the theory of formal multiplication as developed by Berkovitz [1] and Shapiro [23] in $k$-dimensions $(k \geqq 2)$. These results generalize the 1-dimensional results obtained by Rajchman and others [37, pp. 330-344].

Let $S_{1}=\sum a_{m} e^{i(m, x)}$ and $S_{2}=\sum \alpha_{m} e^{i(m, x)}$ be two trigonometric series with the property that for each $m$ the series $\sum_{m}\left|a_{m} \alpha_{n-m}\right|<\infty$. We then define the formal product $S_{3}=S_{1} S_{2}$ to be the trigonometric series

$$
S_{3}=\sum_{m} A_{m} e^{i(m, x)} \quad \text { where } A_{m}=\sum_{m} a_{n} \alpha_{m-n} .
$$


The situation that will occur in the sequel is that the coefficients in $S_{1}$ are "bad" in the sense that they grow large as $|m| \rightarrow \infty$ or at least do not go to zero very rapidly and the coefficients in $S_{2}$ are "good" in the sense that they go to zero very rapidly.

Given a closed domain $\bar{D}$ contained in the interior of $T_{k}$, we shall say $S=\sum_{m} \alpha_{m} e^{i(m, x)}$ is a series in class $\left(S_{\bar{D}}\right)$ if

$$
\begin{aligned}
& \text { for every } \theta>n \text {, there exists an } A \text { depending on } \theta \text { such that } \\
& \left|\alpha_{m}\right| \leqq A(|m|+1)^{-\theta} \quad \text { for all } m \text {; } \\
& \sum_{m} m_{1}^{j 1} \cdots m_{k}^{j_{k}} \alpha_{m} e^{i(m, x)} \\
& =0 \text { for } x \text { in } \bar{D} \text { and } j_{1}, \cdots, j_{k} \text { non-negative integers. }
\end{aligned}
$$

We shall say that $K(x)$ is a spherical harmonic Calderón-Zygmund kernel if

$$
K(x)=P_{n}(x)|x|^{-(n+k)}
$$

where $P_{n}(x)$ is a homogeneous polynomial of degree $n \geqq 1$ and $\Delta P_{n}(x)$ $=0$. It then follows from (71) that

$$
\hat{K}(m)=\gamma(n, k) P_{n}(m)|m|^{-n} \quad \text { for } m \neq 0
$$

where $\gamma(n, k)=(-i)^{n} \Gamma(n / 2) / 2^{k} \Gamma[(n+k) / 2] \pi^{k / 2}$. In this section, we shall deal only with spherical harmonic Calderón-Zygmund kernels.

The following theorem concerning formal multiplication of trigonometric series then prevails (see [1, p. 326 and p. 330], [23, p. 374]).

THEOREM 12. Let $S_{1}=\sum_{m} a_{m} e^{i(m, x)}$ and $S_{2}=\sum_{m} \alpha_{m} e^{i(m, x)}$ where $a_{m}=0(|m| \gamma), \gamma$ an integer $\geqq-(k-1)$, and $S_{2}$ is a series of class $\left(S_{\bar{D}}\right)$, $\bar{D}$ a closed domain contained in the interior of $T_{k}$. Set $S_{3}=S_{1} S_{2}$ $=\sum_{m} A_{m} e^{i(m, x)}$ and $\widetilde{S}_{3}=\sum_{m \neq 0} A_{m} \hat{K}(m) e^{i(m, x)}$ where $K(x)$ is a spherical harmonic Calderon-Zygmund kernel. Then

(i) $S_{3}$ is uniformly $(B-R, \gamma+k-1)$ summable to zero for $x$ in $\bar{D}$.

(ii) $\widetilde{S}_{3}$ is uniformly $(B-R, \gamma+k-1)$ summable for $x$ in $\bar{D}$.

As a corollary to the above theorem and method of proof, we obtain the following generalization of the classical theorem of Riemann (see [1] and [23]):

THEOREM 13. Let $S=\sum_{m} a_{m} e^{i(m, x)}$ where $a_{m}=O(|m| \gamma), \gamma$ an integer $\geqq-(k-1)$. Set $F(x)=\sum_{m \neq 0}(-1)^{\beta}\left(a_{m} /|m|{ }^{2 \beta}\right) e^{i(m, x)}$ where $\beta$ is the integral part of $(k+\gamma) / 2+1$. Suppose that $F(x)$ is class $C^{(2 \beta+k+2)}$ in a domain $D$ contained in $T_{k}$. Then

(i) $\sum_{1 \leq|m| \leq R} a_{m} e^{i(m, x)}\left(1-|m|^{2} / R^{2}\right)^{\gamma+k-1} \rightarrow \Delta^{\beta} F(x)$ as $R \rightarrow \infty$ uniformly in every closed subdomain of $D$, 
(ii) $\sum_{1 \leq|m| \leq R} \hat{K}(m) a_{m} e^{i(m, x)}\left(1-|m|^{2} / R^{2}\right)^{\gamma+k-1} \rightarrow$ finite limit as $R \rightarrow \infty$ uniformly in every closed subdomain of $D$ where $K(x)$ is a spherical harmonic Calderón-Zygmund kernel.

In the proof of the uniqueness theorem for trigonometric series we introduced the notion of generalized Laplacians through the use of averages on $k$-balls. Using averages on $(k-1)$ spheres we can also introduce the notion of generalized Laplacians. (See [22, p. 224].) In particular, we now say that $f(x)$ has a generalized $r$ th-Laplacian at the point $x_{0}$, designated by $\Delta_{r} f\left(x_{0}\right)$, if [using the notation of (70)] $f\left(x_{0}+t \xi\right)$ is in $L^{1}$ on $C(0,1)$ the unit $(k-1)$ sphere in $E_{k}$ for all $t$ sufficiently small and positive and if

$$
\omega_{k-1}^{-1} \int_{C(0,1)} f\left(x_{0}+t \xi\right) d c(\xi)=\sum_{j=0}^{r} \alpha_{j} t^{2 r}+o\left(t^{2 r}\right) .
$$

We then set $\Delta_{j} f\left(x_{0}\right)=2^{2 j} j ! \Gamma(j+k / 2) \alpha_{j} / \Gamma(k / 2)$ for $j=0,1, \cdots, r$.

Using this concept of a generalized $r$ th-Laplacian, Shapiro [22] obtained the following result which is a generalization of the classical theorem concerning trigonometric series and generalized derivatives in 1-dimension [38, p. 69]:

THEOREM 14. Let $S=\sum_{m \neq 0} a_{m} e^{i(m, x)}$ where $a_{m}=o\left(|m|^{\gamma}\right)$, $\gamma \geqq-(k-1)$. A necessary and sufficient condition that there exists an $\alpha \geqq 0$ such that

$$
\lim _{R \rightarrow \infty} \sum_{1 \leq|m| \leq R} a_{m} e^{i(m, x)}\left(1-|m|^{2} / R^{2}\right)^{\alpha}=\beta \text { (finite-valued) }
$$

is that there exists an integer $r>\gamma+k-1$ such that if

$$
F(x)=\sum_{m \neq 0}(-1)^{r}\left(a_{m} /\left.|m|\right|^{2 r}\right) e^{i(m, x)}
$$

then $\Delta_{r} F\left(x_{0}\right)$ exists and is equal to $\beta$.

For a theorem similar to Theorem 14 for Fourier series generalizing a classical theorem of Hardy and Littlewood [38, p. 69] we have the following result of Chandrasekharan's [10]:

THEOREM 15. Let $f(x)$ be in $L^{1}$ on $T_{k}$ and extended by periodicity to all of $E_{k}$, and let $S[f]=\sum_{m} \hat{f}(m) e^{i(m, x)}$. Then a necessary and sufficient condition that there exists an $\alpha \geqq 0$ such that

$$
\lim _{R \rightarrow \infty} \sum_{|m| \leq R} \hat{f}(m) e^{i(m, x)}\left(1-|m|^{2} / R^{2}\right)^{\alpha}=\beta \text { (finite-valued) }
$$

is that there exists $a p>0$ such that 


$$
A(k, p) t^{-k} \int_{B(0, t)} f\left(x_{0}+y\right)\left(1-|y|^{2} t^{-2}\right)^{p-1} d y \rightarrow \beta \quad \text { as } t \rightarrow 0
$$

where $A(k, p)$ is the constant $\left[\omega_{k-1} \int_{0}^{1}\left(1-h^{2}\right)^{p-1} h^{k-1} d h\right]^{-1}$.

7. Geometric integration. In this section, we shall look at some of the applications of multiple trigonometric series to geometric integration theory and potential theory. The future will see many such applications (see the comments in [37, p. 811]).

We shall first discuss an application of multiple trigonometric series to the intrinsic divergence of a vector field. (This result is due to Shapiro [26].)

Let $\mathrm{v}(x)=\left[v_{1}(x), \cdots, v_{k}(x)\right]$ be a continuous vector field defined in a neighborhood of the point $x_{0}$. As before let $B\left(x_{0}, t\right)$ represent the $k$-ball with center $x_{0}$ and radius $t$, let $C\left(x_{0}, t\right)$ represent the $(k-1)$ sphere which is its boundary, let $d c(x)$ represent the natural $(k-1)$ dimensional volume element on $C\left(x_{0}, t\right)$, and let $\mathrm{n}(x)$ represent the outward pointing unit normal vector.

Define

$$
\operatorname{div}^{*} \mathrm{v}\left(x_{0}\right)=\limsup _{t \rightarrow 0}\left|B\left(x_{0}, t\right)\right|^{-1} \int_{C\left(x_{0}, t\right)}[\mathrm{v}(x), \mathrm{n}(x)] d c(x) .
$$

Define $\operatorname{div}_{*} \mathrm{v}\left(x_{0}\right)$ similar using $\lim \inf _{t \rightarrow 0}$. In case $\operatorname{div}^{*} \mathrm{v}\left(x_{0}\right)=\operatorname{div}_{*} \mathrm{v}\left(x_{0}\right)$ is finite, call this common $\operatorname{limit} \operatorname{div} \mathrm{v}\left(x_{0}\right)$. In case, $\operatorname{div} \mathrm{v}\left(x_{0}\right)$ exists, we see that it coincides with the usual definition of the divergence of a vector field defined from an intrinsic point of view. It is furthermore clear that if $\mathrm{v}(x)$ is in class $C^{1}$ in a neighborhood of the point $x_{0}, \operatorname{div} \mathrm{v}\left(x_{0}\right)=\sum_{j=1}^{k} \partial v_{j}\left(x_{0}\right) / \partial x_{j}$.

Let $Q$ be an open $k$-cube in $E_{k}$ and let $\partial Q$ designate its boundary considered as a point set. The divergence theorem in its simplest classical form states that if $\mathrm{v}(x)$ is continuous in $\bar{Q}$, in class $C^{1}$ in $Q$ and if $\operatorname{div} \mathrm{v}(x)$ is in $L^{1}$ on $Q$, then

$$
\int_{\partial Q}[\mathrm{v}(x), \mathrm{n}(x)] d c(x)=\int_{Q} \operatorname{div} \mathrm{v}(x) d x .
$$

Let $Z$ be a closed set of measure zero which lies in $Q$. We define a class $C_{Z}$ of vector fields as follows:

$\mathrm{v}(x)$ is in $C_{Z}$ if

(i) $\mathrm{v}(x)$ is continuous in $\bar{Q}-Z$,

(ii) $\mathrm{v}(x)$ is in $L^{2}$ on $Q$,

(iii) $\operatorname{div}^{*} \mathrm{v}(x)$ and $\operatorname{div}_{*} \mathrm{v}(x)$ are finite in $Q-Z$,

(iv) $\operatorname{div} \mathrm{v}(x)$ exists almost everywhere in $Q$ and is in $L^{1}$ on $Q$. 
Because of (i) and (iv), both the left and right sides of (84) are defined. We shall call $Z$ a negligible set for the divergence theorem, if the divergence theorem holds for every $\mathrm{v}$ in $C_{Z}$, i.e. if $\mathrm{v}(x)$ is in $C_{Z}$, then (84) is true.

In [26], the following result is obtained:

THEOREM 16. A necessary and sufficient condition that $Z$ be a negligible set for the divergence theorem is that $Z$ be of capacity zero.

By capacity zero is meant logarithmic capacity zero in the plane and capacity zero with respect to $|x|^{-(k-2)}$ in $E_{k}$ for $k \geqq 3$.

Condition (ii) above is not artificial because the above theorem is false in $E_{2}$ if we widen $C_{Z}$ by replacing (ii) with "v $(x)$ is in $L^{p}$ on $Q$ with $1 \leqq p<2 . "$ To see this fact, set $\mathrm{v}(x)=\operatorname{grad} \log |x|^{-1}$ in $E_{2}-0$ and take $Q=\left\{x:\left|x_{j}\right|<1, j=1,2\right\}$. Then both sides of (84) are defined but are not equal, and $\mathrm{v}(x)$ is in $L^{p}$ on $Q$ for $1 \leqq p<2$ but is not in $L^{2}$ on $Q$. Furthermore (i), (iii), and (iv) holds with $Z=\{0\}$.

The theorem in multiple trigonometric series which is the key to establishing Theorem 16 is the following [26, p. 611].

Theorem 17. Given $S=\sum_{m} a_{m} e^{i(m, x)}$ where $\bar{a}_{m}=a_{-m}$. Let $Z$ be a closed set of capacity zero contained in the interior of $T_{k}$. Suppose that the following holds:

(i) $\sum_{m \neq 0}\left|a_{m}\right|^{2}|m|^{-2}$ is finite.

(ii) There is a function $F(x)$ continuous in the torus sense in $T_{k}-Z$ and in $L^{1}$ on $T_{k}$ whose Fourier series is given by

$$
S[F]=\sum_{m \neq 0} a_{m}|m|^{-2} e^{i(m, x)} .
$$

(iii) $f^{*}(x)$ and $f_{*}(x)$ are finite in $T_{k}-Z$ and in $L^{1}$ on $T_{k}$ where $f^{*}(x)=\limsup _{t \rightarrow 0} \sum_{m} a_{m} e^{i(m, x)-|m| t}$ and $f_{*}(x)$ is the corresponding $\lim \inf _{t \rightarrow 0}$.

Then $f^{*}(x)=f_{*}(x)$ almost everywhere and $S$ is the Fourier series of $f_{*}(x)$.

Theorem 17 is a $k$-dimensional generalization and extension of a 1-dimensional theorem of Beurling [2]. (For further 1-dimensional results and comments along these lines, see $[38$, p. 194].)

We next look at an application of multiple trigonometric series to the curl of a vector field defined from an intrinsic point of view and obtain a result which can be viewed as a three-dimensional vector analogue of the classical theorem of Rademacher for functions in Lip 1. (See [20] and [36, p. 371].)

Let $\mathrm{v}(x)$ be a three-dimensional vector field defined in a neighborhood of a point $x_{0}$ in $E_{3}$. Let $C_{\mathbf{n}}\left(x_{0}, r\right)$ designate the circle with center 
$x_{0}$ and radius $r$ lying in the plane through $x_{0}$ normal to the unit vector $\mathrm{n}$ and oriented by the usual right hand rule. Define the upper circulation per unit area of $\mathrm{v}$ at $x_{0}$ in the direction $\mathrm{n}$, designated by $D_{\mathrm{n}}^{*} \mathrm{v}\left(x_{0}\right)$, as follows:

$$
D_{\mathrm{nV}}^{*}\left(x_{0}\right)=\limsup _{r \rightarrow 0}\left(\pi r^{2}\right)^{-1} \int_{C_{\mathrm{n}}\left(x_{0}, r\right)}(\mathrm{v}, \mathrm{t}) d s
$$

where $t$ designates the unit tangent vector and $d s$ the differential of arc length. Similarly, define the lower circulation per unit area, $D_{*_{\mathrm{n}} \mathrm{v}}\left(x_{0}\right)$, using $\lim \inf _{r \rightarrow 0}$. If $D_{*_{\mathrm{n}} \mathrm{v}}\left(x_{0}\right)=D_{\mathrm{n}}^{*} \mathrm{v}\left(x_{0}\right)$ and both expressions are finite, designate this common value by $D_{\mathrm{nv}}\left(x_{0}\right)$ and call it the circulation per unit area of $\mathrm{v}$ at $x_{0}$ in the direction $\mathrm{n}$.

The curl of $\mathrm{v}$ is said to exist at $x_{0}$ if $D_{\mathrm{nv}}\left(x_{0}\right)$ exists for every unit vector $\mathrm{n}$ and if, furthermore, there exists a vector $\mathrm{w}$ such that $(\mathrm{w}, \mathrm{n})=D_{\mathrm{n}} \mathrm{v}\left(x_{0}\right)$ for every unit vector $\mathrm{n}$. $\mathrm{w}$ is then called curl $\mathrm{v}\left(x_{0}\right)$.

The curl of $\mathbf{v}$ will be said to exist uniformly at $x_{0}$ if curl $\mathrm{v}\left(x_{0}\right)$ exists and if, furthermore,

$$
\lim _{r \rightarrow 0}\left(\pi r^{2}\right)^{-1} \int_{C_{\mathrm{n}}\left(x_{0}, r\right)}(\mathrm{v}, \mathrm{t}) d s=\mathrm{n} \cdot \operatorname{curl} \mathrm{v}\left(x_{0}\right)
$$

uniformly in $\mathrm{n}$.

It is clear that if $\mathrm{v}(x)$ is in class $C^{1}$ in a neighborhood of the point $x_{0}$, then the curl of $\mathrm{v}$ exists uniformly at the point $x_{0}$. The above definitions are classical and can be found in most of the standard books on advanced calculus or vector analysis.

The following theorem was then established by Shapiro [29]:

THEOREM 18. Let $\mathrm{v}(x)$ be a continuous vector field defined in an open set $R$ contained in $E_{3}$. Suppose there exists three mutually orthogonal unit vectors $\mathrm{e}_{1}, \mathrm{e}_{2}, \mathrm{e}_{3}$ and a constant $K$ such that $\left|D_{\mathrm{e}_{\mathrm{j}}}^{*} \mathrm{v}(x)\right| \leqq K$ and $\left|D_{*_{\mathrm{e}}, \mathrm{v}} \mathrm{v}(x)\right|$ $\leqq K$ for $x$ in $R$ and $j=1,2,3$. Then curl $v$ exists uniformly almost everywhere in $R$.

One of the theorems in multiple trigonometric series on which Theorem 18 depends is the following [26, p. 606]:

TheOREM 19. Let $\mathrm{v}(x)=\left[v_{1}(x), \cdots, v_{k}(x)\right]$ be a continuous periodic real vector field defined in $E_{k}$ with $S\left[v_{j}\right]=\sum_{m} a_{m}^{j} e^{i(m, x)}$. Let $f^{*}(x)$ $=\lim \sup _{t \rightarrow 0} \sum_{m}\left(\sum_{j=1}^{k} i m_{j} a_{m}^{j}\right) e^{i(m, x)-|m| t}$ and let $f_{*}(x)$ be the corresponding $\lim \inf _{t \rightarrow 0}$. Then for every $x$,

$$
\operatorname{div}_{*} \mathrm{v}(x) \leqq f_{*}(x) \leqq f^{*}(x) \leqq \operatorname{div}^{*} \mathrm{v}(x) .
$$

For other applications of the theory of multiple trigonometric 
series to geometric integration theory, we refer the reader to [27] and $[28]$.

We close this survey with the comment that the theory of multiple trigonometric series is yet in its infancy.

\section{Bibliographies.}

\section{Bibliography for SURvey}

1. L. D. Berkovitz, Circular summation and localization of double trigonometric series, Trans. Amer. Math. Soc. 70 (1951), 323-344.

2. A. Beurling, Sets of uniqueness for certain classes of trigonometric series, unpublished lectures of 1953 at the Institute for Advanced Study, Princeton, N. J.

3. S. Bochner, Summation of multiple Fourier series by spherical means, Trans. Amer. Math. Soc. 40 (1936), 175-207.

4. - Boundary values of analytic functions in several variables and of almost periodic functions, Ann. of Math. (2) 45 (1944), 708-722.

5. - Theta relations with spherical harmonics, Proc. Nat. Acad. Sci. U.S.A. 37 (1951), 804-808.

6. - Harmonic analysis and the theory of probability, Univ. of Calif. Press, Berkeley and Los Angeles, 1955.

7. S. Bochner and K. Chandrasekharan, Fourier transforms, Annals of Mathematics Studies, no. 19, Princeton Univ. Press, Princeton, N. J., 1949.

8. A. P. Calderon and A. Zygmund, On the existence of certain singular integrals, Acta Math. 88 (1952), 85-139. 271.

9. - Singular integrals and periodic functions, Studia Math. 14 (1954), 249-

10. K. Chandrasekharan, On the summation of multiple Fourier series. I, Proc. London Math. Soc. 50 (1948), 210-222.

11. K. Chandrasekharan and S. Minakshisundaram, Typical means, Oxford Univ. Press, Oxford, 1952.

12. M. T. Cheng, Uniqueness of multiple trigonometric series, Ann. of Math. (2) 52 (1950), 403-416.

13. A. Erdélyi, W. Magnus, F. Oberhettinger and F. G. Tricomi, Higher transcendental functions, Vol. 2, McGraw-Hill, New York, 1953.

14. H. Helson and D. Lowdenslager, Prediction theory and Fourier series in several variables, Acta Math. 99 (1958), 165-200.

15. K. Hoffman, Banach spaces for analytic functions, Prentice-Hall, Englewood Cliffs, N. J., 1962.

16. O. D. Kellogg, Foundations of potential theory, Ungar, New York, 1929.

17. S. Minakshisundaram, $A$ uniqueness theorem for eigenfunction expansions, Proc. Nat. Acad. Sci. U.S.A. 33 (1947), 76-77.

18. M. Plancherel, Sur l'unicité du développement d'une fonction en sêrie de founctions sphériques, Bull. Sci. Math. 43 (1919), 212-223.

19. W. Rudin, Uniqueness theory for Laplace series, Trans. Amer. Math. Soc. 68 (1950), 287-303.

20. H. Rademacher, Ueber partielle und totale Differenzierbarkeit. I, Math. Ann. 79 (1919), 340-359.

21. T. Rad6, Subharmonic functions, Chelsea, New York, 1949.

22. V. L. Shapiro, Circular summability $C$ of double trigonometric series, Trans. Amer. Math. Soc. 76 (1954), 223-233. 
23. - Localization of conjugate multiple trigonometric series, Ann. of Math. (2) 61 (1955), 368-380.

24. - Generalized Laplacians, Amer. J. Math. 78 (1956), 497-508.

25. - Uniqueness of multiple trigonometric series, Ann. of Math. (2) 66 (1957), 467-480.

26. - The divergence theorem for discontinuous vector fields, Ann. of Math. (2) 68 (1958), 604-624.

27. - Topics in Fourier and geometric analysis, Mem. Amer. Math. Soc. No. $39,1961$.

28. - Symmetric exterior differentiation and flat forms, Canad. J. Math. 14 (1962), 79-86.

29. - The circulation of vector fields, Proc. Amer. Math. Soc. 14 (1963), 818-822.

30. E. M. Stein, On limits of sequences of operators, Ann. of Math. (2) 74 (1961), 140-170.

31. - On certain exponential sums arising in multiple Fourier series, Ann. of Math. (2) 73 (1961), 87-109.

32. E. M. Stein and G. Weiss, On the theory of harmonic functions of several variables, Acta Math. 103 (1960), 25-62.

33. E. C. Titchmarsh, The theory of functions, Oxford Univ. Press, Oxford, 1950.

34. - Introduction to the theory of Fourier integrals, Oxford Univ. Press, Oxford, 1937.

35. G. N. Watson, A treatise on the theory of Bessel functions, Cambridge Univ. Press, Cambridge, 1922.

36. H. Whitney, Geometric integration theory, Princeton Univ. Press, Princeton, N. J., 1957.

37. A. Zygmund, Trigonometric series, Vol. I, Cambridge Univ. Press, Cambridge, 1959. 1959.

38. —, Trigonometric series, Vol. II, Cambridge Univ. Press, Cambridge,

\section{GeNERAL BibliogRAPHY}

This bibliography consists of some of the papers dealing with Fourier series in several variables not referred to in the survey. It is not a complete bibliography of the subject.

101. T. V. Avadhani, On summation over lattice points, J. Indian Math. Soc. 16 (1952), 103-125.

102. L. D. Berkovitz, On double trigonometric integrals, Trans. Amer. Math. Soc. 73 (1952), 345-372. 574.

103. - Double Sturm-Liouville expansions, Duke Math. J. 19 (1952), 567-

104. L. D. Berkovitz and R. P. Gosselin, Restricted summation and localization of double trigonometric series, Duke Math. J. 22 (1955), 243-252.

105. S. Bochner, On spherical partial sums of multiple Fourier series, Rev. Ci. (Lima) 50 (1948), 85-105.

106. - Quasi-analytic functions, Laplace operator, positive kernels, Ann. of Math. (2) 51 (1950), 68-91.

107. - Zeta functions and Green's functions for linear partial differential operators of elliptic type with constant coefficients, Ann. of Math. (2) 57 (1953), 32-56. 

1959.

108. - Lectures on Fourier integrals, Princeton Univ. Press, Princeton, N. J.,

109. S. Bochner and K. Chandrasekharan, On the localization property for multiple Fourier series, Ann. of Math. (2) 49 (1948), 966-978.

110. - Fourier series of $L_{2}$-functions, Duke Math. J. 16 (1949), 579-583.

111. — Lattice points and Fourier expansions, Acta Sci. Math. (Szeged) 12 (1950), 1-15.

112. A. P. Calderón and A. Zygmund, On a problem of Mihlin, Trans. Amer. Math. Soc. 78 (1955), 209-224.

113. - On singular integrals, Amer. J, Math. 78 (1956), 289-309.

114. L. Cesari, Sulle funzioni di due variabli a variazione limitata secondo Tonelli e sulla convergenzu delle relative serie doppie di Fourier, Rend. Sem. Mat. Univ. Rom. (4) 1 (1936), 277-294.

115. - Sulle serie di Fourier funzioni Lipchitziane di piu variabli, Ann. Scuola Norm. Sup. Pisa 7 (1938), 279-295.

116. - Sulle funzioni di piu variabli generalmente a variazione limita e sulla convergenza delle relative serie multiple di Fourier, Pont. Acad. Sci. Comm. 3 (1939), 171-197.

117. K. Chandrasekharan, On Fourier series in several variables, Ann. of Math. (2) 49 (1948), 991-1007.

118. - Fourier series, lattice points, and Watson transforms, Math. Student 19 (1951), 1-11.

119. - On some problems in Fourier analysis, Proc. Internat. Congress Math., 1954, Amsterdam, vol. III, pp. 85-91, Noordhoff, Groningen; North-Holland, Amsterdam, 1956.

120. K. Chandrasekharan and S. Minakshisundaram, Some results on double Fourier series, Duke Math. J. 14 (1947), 731-753.

121. V. G. Chelidze, On the absolute convergence of double Fourier series, C.R. (Doklady) Acad. Sci. URSS (N.S.) 54 (1946), 117-120.

122. M. T. Cheng, Some Tauberian theorems with applications to multiple Fourier series, Ann. of Math. (2) 50 (1949), 763-776.

123. - The Gibbs phenomenon and Bochner's summation method. II, Duke Math. J. 17 (1950), 477-490.

124. G. Cross, Multiple trigonometric series of a particular type, Duke Math. J. 29 (1962), 489-495.

125. K. de Leeuw and I. Glicksberg, Quasi-invariance and analyticity of measures on compact groups, Acta Math. 109 (1963), 179-205.

126. H. Geiringer, Trigonometrische Doppelreihen, Monatsh. Math. Phys. 29 (1918), 65-144.

127. J. J. Gergen, Convergence criteria for double Fourier series, Trans. Amer. Math. Soc. 35 (1933), 29-63.

128. R. P. Gosselin, On the theory of localization for double trigonometric series, Ann. Soc. Polon. Math. 24 (1951), 49-77.

129. G. Grunwald, Zur Summabilitäs theorie der Fourier Doppelreihe, Proc. Cambridge Philos. Soc. 35 (1939), 343-350.

130. G. H. Hardy, On double Fourier series and especially those which represent the double Zeta function with real and incommensurable parameters, Quart. J. Math. 37 (1906), 53-79.

131. H. Helson, Conjugate series in several variables, Pacific J. Math. 8 (1958) 437446. 
132. H. Helson and D. Lowdenslager, Prediction theory and Fourier series in several variables. II, Acta Math. 106 (1961), 175-212.

133. J. G. Herriot, Nörlund summability of multiple Fourier series, Duke Math. J. 11 (1944), 735-754.

134. - Partial couplings for double Fourier series, Duke Math. J. 19 (1952), 183-198.

135. C. S. Herz, On the number of lattice points in a convex set, Amer. J. Math. 84 (1962), 126-133.

136. - Fourier transforms related to convex sets, Ann. of Math. (2) 75 (1962), 81-92.

137. E. W. Hobson, The theory of functions of a real variable, vol. 2, Cambridge Univ. Press, Cambridge, 1927.

138. J. Horvath, Sur les fonctions conjuguées d plusieurs variables, Nederl. Akad. Wetensch. Proc. Ser. A. 56 = Indag. Math. 15 (1953), 17-29.

139. L. Lichtenstein, Über das Porssonsche Integral, J. Reine Angew. Math. 141 (1912), 12-42.

140. J. Marcinkiewicz and A. Zygmund, On the summability of double Fourier series, Fund. Math. 32 (1939), 112-132.

141. I. V. Matveev, On summation of double Fourier series of functions of two variables, Uspehi Mat. Nauk 12 (1957), 221-229.

142. S. Minakshisundaram, Notes on Fourier expansions. III, Amer. J. Math. 71 (1949), 60-66.

143. S. Minakshisundaram and $O$. Szasz, On absolute convergence of multiple Fourier series, Trans. Amer. Math. Soc. 61 (1947), 36-53.

144. J. Mitchell, On double Sturm-Liouville series, Amer. J. Math. 65 (1943), 616636.

145. - On the spherical convergence of multiple Fourier series, Amer. J. Math. 73 (1951), 211-226.

146. C. N. Moore, On the summability of double Fourier series of discontinuous functions, Math. Ann. 74 (1913), 555-572.

147. M. Morse and W. Transue, The Frechet variation and the convergence of multiple Fourier series, Proc. Nat. Acad. Sci. U.S.A. 35 (1949), 395-399.

148. - The Frechet variation and a generalization for multiple Fourier series of the Jordan test, Riv. Mat. Univ. Parma 1 (1950), 3-18.

149. J. P. Natanson, On convergence of certain interpolation processes for functions of two arguments, Mat. Sb. (N.S.) 33(75) (1953), 219-232.

150. W. Nowacki, Fourier sythesese von Kristallen und ihre anwendung in der Chemie, Verlag Birkhäuser, Basel, 1952.

151. G. E. Reves and O. Szasz, Some theorems on double trigonometric series, Duke Math. J. 9 (1942), 693-705.

152. V. L. Shapiro, Square summation and localization of double trigonometric series, Amer. J. Math. 75 (1953), 347-357.

153. - A note on the uniqueness of double trigonometric series, Proc. Amer. Math. Soc. 4 (1953), 692-695.

154. - Logarithmic capacity of sets and double trigonometric series, Canad. J. Math. 6 (1954), 582-592.

155. - Summability and uniqueness of double trigonometric integrals, Trans. Amer. Math. Soc. 77 (1954), 322-339.

156. - Generalized Laplacians of the second kind and double trigonometric series, Duke Math. J. 21 (1954), 173-178. 
157. - Cantor-type uniqueness of multiple trigonometric integrals, Pacific J. Math. 5 (1955), 607-622. 444.

158. — , The Laplacian of Fourier transforms, Duke Math. J. 22 (1955), 435-

159. K. Sokol-Solowski, On trigonometric series conjugate to Fourier series in two variables, Fund. Math. 34 (1947), 166-182.

160. E. M. Stein, Functions of exponential type, Ann. of Math. (2) 65 (1957), 582592.

161. - Localization and summability of multiple Fourier series, Acta Math. 100 (1958), 93-147.

162. G. I. Sunouchi, On double Fourier series, Proc. Amer. Math. Soc. 1 (1950), $522-525$.

163. L. Tonelli, Serie trigonometriche, Zanichelli, Bologna, 1928.

164. - Sulle serie doppe di Fourier, Ann. Scuola Norm. Sup. Pisa 6 (1937), 315-326.

165. A. J. White, On the summability, by spherical Riesz means, of multiple Fourier series, J. London Math. Soc. 35 (1960), 495-502.

166. - On the summability, spherical Riesz means, of derived multiple Fourier series, Amer. J. Math. 83 (1961), 602-622.

167. I. E. Zak, On some properties of conjugate double trigonometric series, Dokl. Akad. Nauk SSSR 73 (1950), 5-8.

168. A. Zygmund, On the summability of multiple Fourier series, Amer. J. Math. 69 (1947), 836-850.

169. A. Zygmund, W. Transue, M. Morse, A. P. Calderon and S. Bochner, Contributions to Fourier analysis, Princeton Univ. Press, Princeton, N. J., 1950.

UNIVERSITY OF OREGON 